\title{
Robust one dimensionality at twin grain boundaries in $\mathrm{MoSe}_{2}$
}

\author{
T. Čadež, ${ }^{1,2}$ L. Li, ${ }^{3}$ E. V. Castro, ${ }^{1,2,4,5}$ and J. M. P. Carmelo ${ }^{2,6,7,8}$ \\ ${ }^{1}$ Beijing Computational Science Research Center, Beijing 100193, China \\ ${ }^{2}$ Center of Physics of University of Minho and University of Porto, P-4169-007 Oporto, Portugal \\ ${ }^{3}$ Department of Physics, National University of Singapore, Singapore 117551, Republic of Singapore \\ ${ }^{4}$ Departamento de Física e Astronomia, Faculdade de Ciências, Universidade do Porto, 4169-007 Porto, Portugal \\ ${ }^{5}$ CeFEMA, Instituto Superior Técnico, Universidade de Lisboa, Avenida Rovisco Pais, 1049-001 Lisboa, Portugal \\ ${ }^{6}$ Department of Physics, University of Minho, Campus Gualtar, P-4710-057 Braga, Portugal \\ ${ }^{7}$ Boston University, Department of Physics, 590 Commonwealth Ave., Boston, Massachusetts 02215, USA \\ ${ }^{8}$ Massachusetts Institute of Technology, Department of Physics, Cambridge, Massachusetts 02139, USA
}

(Received 25 August 2018; published 3 April 2019)

\begin{abstract}
We show that $1 \mathrm{D}$ electron states confined at twin grain boundaries in $\mathrm{MoSe}_{2}$ can be modeled by a three-orbital tight-binding model including a minimum set of phenomenological hopping terms. The confined states are robust to the details of the defect hopping model, which agrees with their experimental ubiquity. Despite a valley Chern number which is finite and opposite on both sides of the defect, there is no topological protection of the confined states. This turns out to be an essential feature to have only one confined electronic band, in agreement with experiments, instead of two, as the bulk-edge correspondence would imply. Modeling the confined state as a 1D interacting electronic system allows us to unveil a mobile quantum-impurity-type behavior at energy scales beyond the Tomonaga-Luttinger liquid with an interaction range which extends up to the lattice spacing, in excellent agreement with angle-resolved photoemission spectroscopy measurements.
\end{abstract}

DOI: 10.1103/PhysRevB.99.155109

\section{INTRODUCTION}

One-dimensional (1D) electronic systems are the host of many interesting phenomena, including the possible condensed-matter realization of Majorana zero modes due to the nontrivial topology of the electron states [1], the observation, due to electron correlations [2], of both low-energy Tomonaga-Luttinger liquid (TLL) physics and higher-energy mobile quantum impurity model (MQIM) behavior, beyond TLL [3], as well as the observation of spin and charge separation at all energy scales [4], to mention a few. In a 3D world, one-dimensionality is obviously not the rule. Fortunately, a variety of examples can be found in nature (or synthesized) carbon nanotubes are a paradigmatic example [5], but also semiconducting nanowires such as, for example, InSb and InAs [1,6], and assembled atom chains on surfaces [2,7] have been in the spotlight recently, with prominent technological potential in some cases.

The advent of 2D materials [8], in particular the realization of a class known as semiconducting transition metal dichalcogenides (TMDs) [9], formula $M \mathrm{X}_{2}$, where $M$ is a transition metal (e.g., Mo, W) and $\mathrm{X}$ is a chalcogen (e.g., $\mathrm{S}, \mathrm{Se})[10,11]$, allowed for a distinct type of 1D electron system: a confined state at the twin-grain-boundary (TGB) defect shown in Fig. 1(a). The presence of such 1D states inside the bulk gap, in excess of $1 \mathrm{eV}$, has been clearly demonstrated experimentally [12-15]. Their metallicity also became apparent, as well as intrinsic 1D behavior such as a Peierls transition originating a charge-density-wave order below $T \lesssim$ $250 \mathrm{~K}$, as well as spin and charge separation characteristic of a correlated 1D system $[4,14]$. In this paper, we show that the three-orbital tight-binding (TB) model of Ref. [16], widely used to describe physics around the gap edges in TMDs, can be used to describe the confined 1D states at TGBs. A minimum set of phenomenological hoppings are included to couple the two sides of the TGB. The induced in-gap states are robust to the details of the defect hopping model, being present in its simplest version where only nearest-neighbor (NN) hoppings between $d_{z^{2}}$ orbitals are allowed. The respective spectrum is shown in Fig. 1(b), where a band of states localized at the TGB is clearly seen crossing the gap. The localized nature of the states is depicted in Fig. 1(c), where we show the probability density for a $K$-valley state. The valley Chern number, which changes signs across the boundary and takes values $C_{v}= \pm 1$, does not warrant topological protection of the 1D states. This is crucial to stabilize a single band at the TGB, in agreement with experiments and ab initio simulations $[14,17,18]$, as opposed to what would be implied by the Chern number change $\left|\Delta C_{v}\right|=2$ across the TGB [19]. The stability of the single band is, however, reminiscent of the Berry phase difference between the two sides of the TGB [20].

Including interactions in the effective 1D system, and explicitly accounting for the effects of the finite range of the interaction between the MQIM charge degrees of freedom, improves the agreement with angle-resolved photoemission spectroscopy (ARPES) experiments beyond that reached in Ref. [4].

The paper is organized as follows: In Sec. II, we introduce the TB model used to describe the electronic properties of the TGB. The continuum theory valid on both sides of the line defect is discussed in Sec. III, where we also provide 
(a)
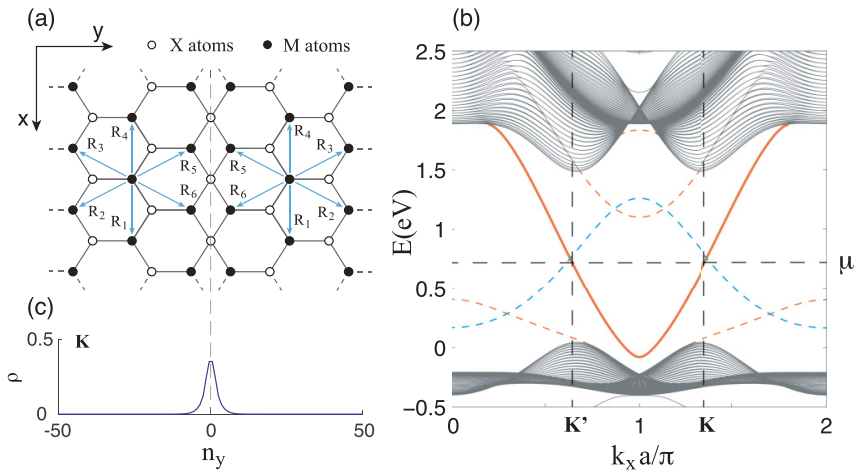

FIG. 1. (a) $\mathrm{MX}_{2}$ lattice with a TGB defect along the $x$ direction. (b) Spectrum for a ribbon of $\mathrm{MX}_{2}$ with a TGB in the middle, obtained with a single hopping parameter to couple the two sides of the TGB (see text). A band of electron states confined at the TGB is shown as a thick (orange) line. Thin (black) lines are bulk states and short dashed (blue and orange) are other 1D states. (c) Probability density for an electron confined at the TGB in the $K$-valley.

a detailed topological analysis. The effect of the electron finite-range interactions within the line defects is studied in Sec. IV. In Sec. V, the key results are summarized and some conclusions are drawn. We also include two appendices: In Appendix A, we derive the continuum theory; in Appendix B, some expressions useful for the discussion of the electron finite-range interactions associated with metallic states in the line defects are provided.

\section{TIGHT-BINDING ANALYSIS}

We model electrons in $\mathrm{MoSe}_{2}$ using an $M$ atom threeorbital NN-TB Hamiltonian given by

$$
H_{0}=\sum_{i, \alpha} \sum_{\gamma, \gamma^{\prime}, \sigma} c_{i, \gamma, \sigma}^{\dagger} E_{\gamma, \gamma^{\prime}}^{\sigma}\left(\mathbf{R}_{\alpha}\right) c_{i+\mathbf{R}_{\alpha}, \gamma^{\prime}, \sigma},
$$

where $c_{i, \gamma, \sigma}^{\dagger}$ is an electron creation operator on lattice site $i, M$-atom orbital $\gamma=d_{z^{2}}, d_{x y}, d_{x^{2}-y^{2}}$, spin $\sigma=\uparrow, \downarrow$, and $\mathbf{R}_{\alpha}$ with $\alpha=1, \ldots, 6$ are the six vectors connecting NN atoms as shown in Fig. 1(a). $E_{\gamma, \gamma^{\prime}}^{\sigma}\left(\mathbf{R}_{\alpha}\right)$ are hopping integrals as given in Ref. [16] for the NN model [21]. We write the TB Hamiltonian, including the TGB, as

$$
H=H_{\mathrm{L}}+H_{\mathrm{R}}+H_{\mathrm{TGB}},
$$

with $H_{\mathrm{L}} \equiv H_{0}$ to the left of the TGB $(y<0)$ and $H_{\mathrm{R}} \equiv$ $\sigma_{v}^{\dagger} H_{0} \sigma_{v}$ to the right $(y>0)$, where $\sigma_{v}$ is the reflection operator associated to the mirror transformation $y \rightarrow-y$ [see Fig. 1(a)], and $H_{\mathrm{TGB}}$ couples left and right regions. $H_{\mathrm{R}}$ can be written as in Eq. (1) with the NN hoppings reversed [see Fig. 1(a)], so that the total Hamiltonian in Eq. (2) respects the apparent mirror symmetry of the system with respect to the line defect. $H_{\mathrm{TGB}}$ is modeled in two ways: a simplified model, where only the NN hopping between $M$-atom $d_{z^{2}}$ orbitals is allowed, and a more elaborated model, where three NN hopping terms are allowed across the TGB.

The results for the simplified model are shown in Figs. 2(a)-2(c), respectively, for hopping values $\left|\tilde{t}_{z^{2}}\right|=$ $0.2,0.6,1.0 \mathrm{eV}$, where we considered a ribbon with translational invariance along the $x$ direction and $N_{y}=100$ unit
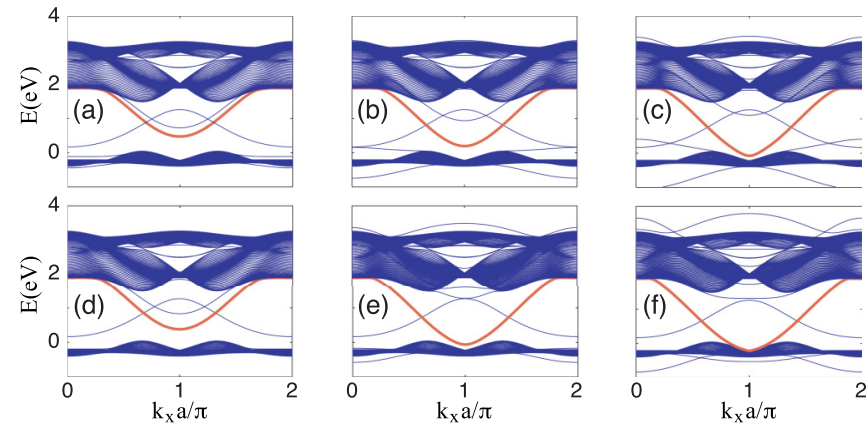

FIG. 2. Energy spectrum for a ribbon with a TGB in the middle, obtained using the TB model with (a)-(c) only one NN hopping between $d_{z^{2}}$ orbitals, $\left|\tilde{t}_{z^{2}}\right|=0.2,0.6,1.0 \mathrm{eV}$, respectively; (d)-(e) three NN hoppings involving $d_{z^{2}}$ and $d_{x^{2}-y^{2}}$ (see main text), where $\left|\tilde{t}_{z^{2}, x^{2}-y^{2}}\right|=0.2,0.6,1.0 \mathrm{eV}$, respectively.

cells in the $y$ direction, transverse to the TGB. Figure 2(c) is the same as the one in Fig. 1(c). In the latter, the dashed blue line corresponds to edge states localized at the outer edges of the ribbon, so-called $M$ edges. These edge states, present in all panels of Fig. 2, have been studied elsewhere $[16,22,23]$ and will be ignored here. In the limit $\tilde{t}_{z^{2}}=0$, the TGB is composed of two uncoupled $X$ edges, which also support edge states [24,25]. In Fig. 2(a), it is seen that a finite $\tilde{t}_{z^{2}}$ lifts the degeneracy of the two $X$-edge states. On increasing $\tilde{t}_{z^{2}}$ [Figs. 2(b) and 2(c)], bonding and antibonding states are formed. The bonding state is pushed down in energy, particularly when the localization length is smaller $\left(k_{x} a \simeq \pi\right)$, and will be partially occupied.

$A b$ initio calculations clearly show that the in-gap states localized at the TGB are derived from $M$-atom orbitals $[18,26]$. Within the three-orbital NN-TB model adopted here, we have verified that including hoppings involving the orbital $d_{x y}$ has little effect on the dispersion of in-gap states, implying that the orbitals $d_{z^{2}}$ and $d_{x^{2}-y^{2}}$ are the most important for the defect state. With this in mind, we developed a more realistic model for $H_{\mathrm{TGB}}$ considering three hoppings across the defect: direct hoppings $\tilde{t}_{z^{2}}$ and $\tilde{t}_{x^{2}-y^{2}}$, and a crossed term $\tilde{t}_{z^{2}, x^{2}-y^{2}}$. To reduce the number of free parameters, we fix the hopping ratios to the values in the bulk, $\tilde{t}_{z^{2}}: \tilde{t}_{x^{2}-y^{2}}:-\tilde{t}_{z^{2}, x^{2}-y^{2}}=t_{z 2}^{\text {bulk }}: t_{x^{2}-y^{2}}^{\text {bulk }}$ : $t_{z^{2}, x^{2}-y^{2}}^{\text {bulk }}$. The minus sign in $\tilde{t}_{z^{2}, x^{2}-y^{2}}$ accounts for the $\pi / 2$ rotation of the hopping direction with respect to $\mathbf{R}_{1}$, which is the reference for the hopping amplitudes in the bulk [16]. Figures 2(d)-2(f) show the spectrum for increasing values of $\left|\tilde{t}_{z^{2}, x^{2}-y^{2}}\right|=0.2,0.6,1.0 \mathrm{eV}$. The results are very similar to those obtained with the single-hopping model. Allowing for hoppings involving the $d_{x y}$-orbital does not significantly change the results, which agrees with $d_{x y}$ minor role in TGB states.

In both models, we allowed for hopping values $t \sim 1 \mathrm{eV}$. These are higher then bulk values [16], as a consequence of the shorter NN distance between $M$ atoms on opposite sides of the TGB (20\% smaller [26]). We have deliberately ignored spin-orbit coupling (SOC) since TGB states derive from the $X$-edge states, which are weakly affected by SOC. Intrinsic SOC can be easily incorporated [27-29], but only at very low temperatures will the spin-degeneracy assumption breakdown. 


\section{CONTINUUM THEORY AND TOPOLOGICAL CONSIDERATIONS}

\section{A. Low-energy two-band model}

A continuum theory describing the left $(y<0)$ and right $(y>0)$ regions [see Fig. 1(a)] can be derived from the threeorbital TB model (see Appendix A). The Hamiltonian reads

$$
\mathcal{H}_{\tau \mu}(\boldsymbol{q})=v \hbar\left(\tau q_{x} \sigma_{x}+\mu q_{y} \sigma_{y}\right)+\left(\Delta+\beta q^{2}\right) \sigma_{z}+\epsilon_{\mathrm{F}} \sigma_{0},
$$

where $\boldsymbol{q}=\boldsymbol{k}-\tau \boldsymbol{K}$ is the small momentum with respect to valley $K(\tau=+1)$ or $K^{\prime}(\tau=-1), \mu=+1$ on the left $(y<0)$ and $\mu=-1$ on the right $(y>0)$ regions, and $\epsilon_{\mathrm{F}}$ is the chemical potential. The Pauli matrices $\sigma_{i=x, y, z}$ act on the space of conduction and valence band states at $\tau \boldsymbol{K}$, with $\sigma_{0}$ for the identity. For $\mathrm{MoSe}_{2}$, the coefficients take the values $v \simeq 5.6 \times 10^{5} \mathrm{~ms}^{-1}, 2 \Delta \simeq 1.44 \mathrm{eV}, \beta \simeq-3.01 \mathrm{eV} \AA^{2}$, and $\epsilon_{\mathrm{F}} \simeq 0.76 \mathrm{eV}$. Apart from SOC, we are ignoring electron-hole asymmetry and trigonal warping terms, which have much smaller coefficients (see Appendix A).

Equation (3) can be cast in the form

$$
\mathcal{H}_{\tau \mu}(\boldsymbol{q})=\boldsymbol{h}(\boldsymbol{q}) \cdot \boldsymbol{\sigma}+\epsilon_{\mathrm{F}} \sigma_{0},
$$

where $\sigma$ is the vector of Pauli matrices. Equation (4) allows for straightforward topological analysis in terms of the valley Chern number, as done in the following.

\section{B. Chern number}

Within the two-band continuum theory of the previous section, the valley Chern number is defined by $C_{\tau, \mu}^{v}=$ $\frac{1}{2 \pi} \int_{-\infty}^{\infty} \int_{-\infty}^{\infty} \Omega_{\tau, \mu}^{v}(\boldsymbol{q}) d q_{x} d q_{y}$, with $\Omega_{\tau, \mu}^{v}$ the Berry curvature for the lower band [30,31],

$$
\Omega_{\tau, \mu}^{v}(\boldsymbol{q})=\frac{1}{2} \frac{\partial \boldsymbol{h}}{\partial q_{x}} \times \frac{\partial \boldsymbol{h}}{\partial q_{y}} \cdot \frac{\boldsymbol{h}}{h^{3}},
$$

with the vector $\boldsymbol{h}(\boldsymbol{q})$ as in Eq. (4). After integration [32], we obtain

$$
C_{\tau, \mu}^{v}=\frac{1}{2} \tau \mu[\operatorname{sign}(\Delta)-\operatorname{sign}(\beta)] .
$$

The dependence on $\Delta$ and $\beta$ is known [33,34]: for $\Delta>0$ and $\beta<0$, the case of TMDs, the system is topologically nontrivial with $C_{\tau, \mu}^{v}=\tau \mu$. The dependence on the valley index $\tau$ is required by time-reversal symmetry. The dependence on $\mu$, which accounts for the position, left or right, with respect to the TGB, needs clarification.

For a Chern number change $\Delta C_{\tau}^{v}=\left|C_{\tau,+1}^{v}-C_{\tau,-1}^{v}\right|=$ 2 , we would expect two chiral modes per valley (per spin) running along the boundary, as implied by the bulkedge correspondence [19]. These modes appear as bound states of the Hamiltonian $\mathcal{H}_{\tau \mu}\left(q_{x}, y\right)$, obtained from Eq. (3) with $q_{y} \rightarrow-i \partial_{y}$ and $\mu \rightarrow \mu(y)$, where $\mu(y<0)=+1$ and $\mu(y>0)=-1$. Close inspection shows that no bound-state solution exists, contrary to other $2 \mathrm{D}$ systems with domain walls [35-37]. This is consistent with the absence of a gap closing associated with a change of sign in $\mu$ (the spectrum $E_{\boldsymbol{q}}=\epsilon_{\mathrm{F}} \pm|\boldsymbol{h}(\boldsymbol{q})|$ is independent of $\mu$ ). The apparent discrepancy stems from the transformation $y \rightarrow-y$ relating left and right regions, which implies a reversal of the chirality of edge states, and thus a sign change of the valley Chern number [38],

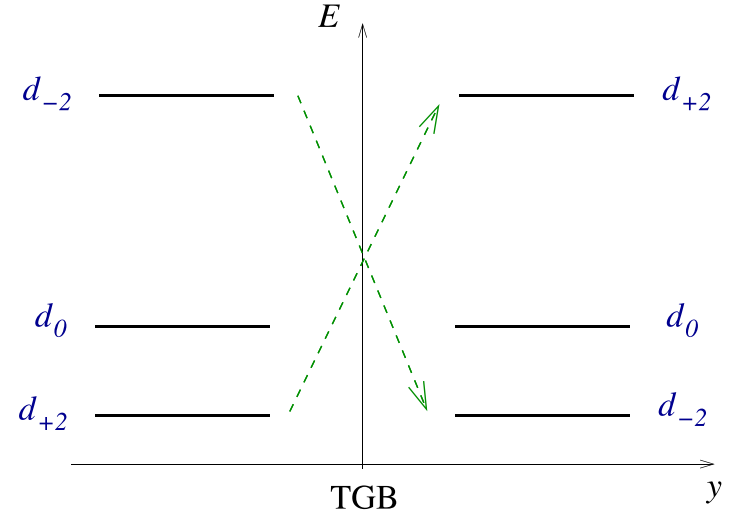

FIG. 3. Sketch of the band structure right at a single valley momentum on both sides of the TGB. A band inversion involving the highest and the lowest Bloch states $d_{ \pm 2}$ is apparent.

but not a gap closing. The lack of topological protection, in contrast to that found in topological band insulators [39], is crucial to make our TB results compatible with experiments. Indeed, for a filling $n=2 / 3$ (including spin), expected for the charge neutral system, a single 1D band-and not twocrossing the Fermi level was observed [4]. The stability of these states can be linked to the 1D Berry phase difference between the two sides of the TGB [20], as discussed in Sec. III D.

\section{Low-energy three-band theory}

The inadequacy of the two-band theory of Sec. III A to describe bound states at the TGB can be understood within a continuum three-band approximation. Such an approximation may be obtained by writing the momentum-space version of $H_{0}$ defined in Eq. (1), and expanding around the corners of the Brillouin zone (BZ). As is well known, right at the corner momenta $\boldsymbol{k}=\tau \boldsymbol{K}(\tau= \pm 1)$, we obtain Bloch states with welldefined $z$-component orbital angular momentum [16],

$$
\begin{aligned}
\left|\tau \boldsymbol{K}, d_{0}\right\rangle & =\left|\tau \boldsymbol{K}, d_{z^{2}}\right\rangle, \\
\left|\tau \boldsymbol{K}, d_{+2 \tau}\right\rangle & =\frac{1}{\sqrt{2}}\left[\left|\tau \boldsymbol{K}, d_{x^{2}-y^{2}}\right\rangle+i \tau\left|\tau \boldsymbol{K}, d_{x y}\right\rangle\right], \\
\left|\tau \boldsymbol{K}, d_{-2 \tau}\right\rangle & =\frac{1}{\sqrt{2}}\left[\left|\tau \boldsymbol{K}, d_{x^{2}-y^{2}}\right\rangle-i \tau\left|\tau \boldsymbol{K}, d_{x y}\right\rangle\right],
\end{aligned}
$$

with momentum states $\left|\boldsymbol{k}, d_{\gamma}\right\rangle \equiv c_{\boldsymbol{k}, \gamma}^{\dagger}|0\rangle$ dual to the $c_{i, \gamma}^{\dagger}|0\rangle$ states in Eq. (1).

In the presence of the TGB, a low-energy three-band model can be invoked far away from the line defect. On the $y<0$ side, Eq. (2) reduces to $H_{\mathrm{L}}$, while on the $y>0$ only $H_{\mathrm{R}}$ matters. Since $H_{\mathrm{L}} \equiv H_{0}$ and $H_{\mathrm{R}}$ is related to $H_{0}$ through a $y \rightarrow$ $-y$ mirror transformation, we can show that the three Bloch states given in Eqs. (7) are eigenstates on both sides of the TGB right at the corner momenta $\boldsymbol{k}=\tau \boldsymbol{K}$ (see Appendix A for details). However, the eigenergies are different, with a gap inversion affecting the two states $d_{+2 \tau}$ and $d_{-2 \tau}$ [compare Eqs. (A10) and (A19)]. Such gap inversion is sketched in Fig. 3.

The origin of the confined 1D states at the TGB may be traced back to the gap inversion involving the valence and the 


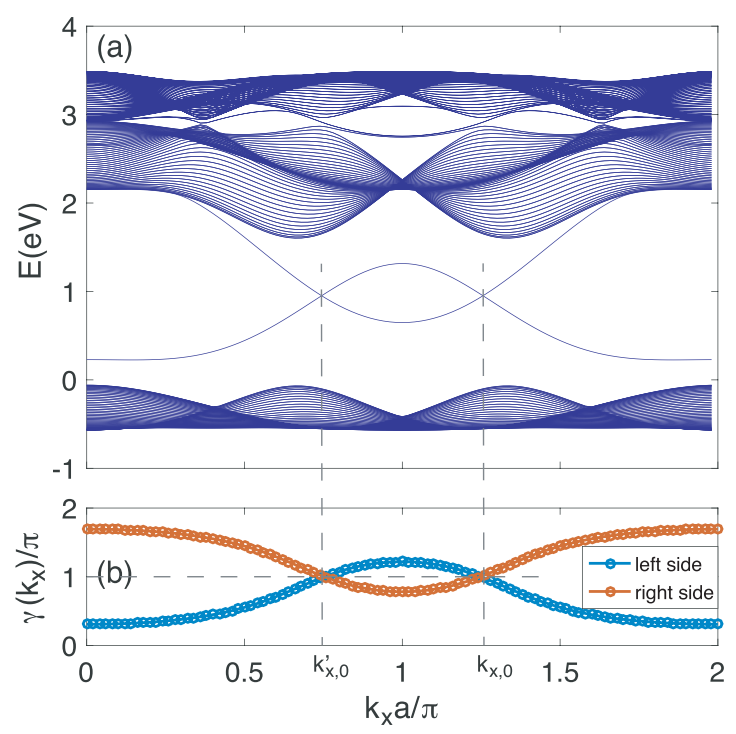

FIG. 4. (a) The spectrum of the three-band model of TMDs under open boundary condition along $y$ and (b) the corresponding Berry phase $\gamma\left(k_{x}\right)$. The blue and red colors in (b) are for the two halves with $y<0$ and $y>0$, respectively, in the system with TGB defect as described in Fig. 1(a).

highest bands described by the three-orbital TB model (see Fig. 3). A low-energy two-band approximation, where only the two lowest energy states are considered, cannot capture this effect. This picture also provides an understanding for why SOC effects are not important, as these amount just to a small variation of the band energies, not affecting the gap inversion.

\section{Berry phase}

The state localized at the TGB is topologically originated at the difference of Berry phase across the boundary [20].

The $X$ - and $M$-edge states localize at the boundary along $x$, therefore the system can be viewed as 1D lattice periodically modulated by a parameter $k_{x}$, and the edge states can be described by a Berry phase defined as

$$
\gamma\left(k_{x}\right)=i \oint d k_{y}\left\langle u_{k_{x}, k_{y}}\left|\partial_{k_{y}}\right| u_{k_{x}, k_{y}}\right\rangle=\pi,
$$

with $u_{k_{x}, k_{y}}$ the occupied state at $1 / 3$ filling (without spin), obtained by diagonalizing Eq. (A2) [40]. A topologically nontrivial $1 \mathrm{D}$ insulating system is generally characterized by a $\pi$ Berry phase, and has a pair of topologically protected degenerate edge states localized at the two ends of the 1D chain. However, the $X$ - and $M$-edge states of TMDs have different energy dispersions versus $k_{x}$, and $\gamma\left(k_{x}\right)$ also varies continuously.

In Fig. 4, we illustrate the spectrum as a function of $k_{x}$ under open boundary condition along $y$, and the corresponding Berry phase $\gamma\left(k_{x}\right)$. The two edge states in Fig. 4(a) become degenerate at two certain values of $k_{x}=k_{x, 0}$ (and $\left.k_{x, 0}^{\prime}\right)$, corresponding to a Berry phase $\gamma\left(k_{x, 0}\right)=\pi$. The Berry phase varies continuously away from $k_{x, 0}$, thus the degeneracy of edge states is lifted. Nevertheless, the edge states can be interpreted as a continuation of the degenerate edge states at $k_{x, 0}$, and thus topologically originated at the $\pi$ Berry phase.
In the system with TGB that we consider in this paper, the two sides with $y<0$ and $y>0$ correspond to the Berry phase $\gamma\left(k_{x}\right)$ and $-\gamma\left(k_{x}\right)$, respectively, and the state localized at the TGB can be associated with the difference of the Berry phase across the TGB [20].

\section{EFFECT OF CORRELATIONS WITHIN THE LINE DEFECTS}

Consistent with the robust 1D nature of the metallic states in $\mathrm{MoSe}_{2}$ line defects found here, the approach used by Ma et al. [4] for a class of 1D correlated electronic lattice systems whose finite-range potential general properties are reported below that applies to such states is a particular case of the general MQIM [3]. It uses a representation in terms of charge and spin particles that emerge in such systems at all energy scales. The main effects of the electron repulsion between different sites are within that approach in the interaction of the charge particles with the charge- or spin-hole mobile impurity created under one-electron removal excitations.

For the $\mathrm{MoSe}_{2}$ line defects, the effective range $R_{\text {eff }}$ of the latter interaction is small. Consistently, the studies of Ma et al. [4] used $R_{\text {eff }}=0$. Here we account for the effects of higherorder charge-particle phase-shift terms that contain $R_{\text {eff }}$. We find that for the $\mathrm{MoSe}_{2}$ line defects, $R_{\text {eff }}$ is of about one lattice spacing $a_{0}$. We confirm that using $R_{\text {eff }} \approx a_{0}$ or $R_{\text {eff }} \approx 0$ leads to theoretical predictions for such line defect ARPES peaks distribution within the experimental uncertainty. However, we find that accounting for the higher-order charge-particle phase-shift terms and thus using $R_{\text {eff }} \approx a_{0}$ improves the agreement with experiments.

In this section, we generally use units of lattice spacing $a_{0}$ one and Planck constant $\hbar$ one so that wave vectors are called momenta.

\section{A. The MQIM for finite-range interactions}

A decisive low-energy property of 1D metallic correlated systems is the low-energy power-law suppression of the density of states (SDS) at the Fermi level. The experimental value of the corresponding power-law SDS exponent $\alpha$ is typically equal to or larger than $1 / 2$ [2,4,41]. Figure 5 displays the SDS of $\mathrm{MoSe}_{2}$ line defects close to the Fermi level measured at room temperature (to avoid charge-density-wave transition) and corresponding analytical lines for SDS power-law exponent $\alpha=0.70, \alpha=0.75$, and $\alpha=0.80$.

It is known that the SDS exponent is such that $\alpha<1 / 8$ for the integrable correlated electronic models such as the 1D Hubbard model (1DHM) with on-site repulsion $U$ and transfer integral $t$ whereas an $\alpha>1 / 8$ stems from finite-range electron interactions in nonintegrable models [42] whose range is at least of one lattice spacing.

According to the principle of emergence, the properties of a physical system are mainly determined by how electrons are organized in it [43]. In the case of the correlated electronic systems to which the MQIM applies [3], such an organization gives rise to emerging fractionalized particles whose phase shifts are imposed by mobile quantum impurities created under transitions to excited states.

The MQIM scheme used in the studies of Ref. [4] accounted for the leading-order term of an effective-range 


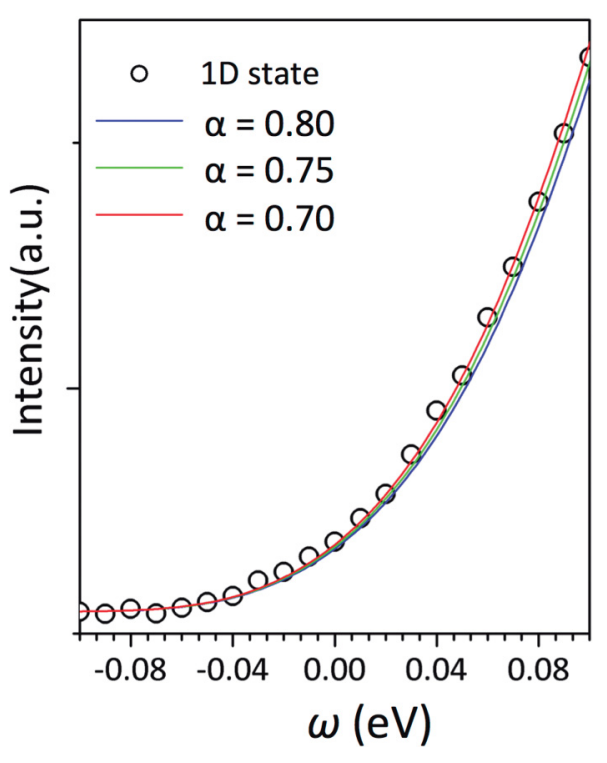

FIG. 5. The suppression of the density of states of mirror twin grain boundaries in monolayer $\mathrm{MoSe}_{2}$ close to the Fermi level measured at room temperature and corresponding theoretical predicted power-law lines for $\alpha=0.70, \alpha=0.75$, and $\alpha=0.80$. It is obtained by plotting the angle-integrated photoemission intensity as a function of binding energy $\omega$. The experimental data are well fit for $\alpha=$ $0.75 \pm 0.5$ and thus with a corresponding uncertainty estimated to be as large as \pm 0.05 . Source: Fig. 4(c) of Ma et al. [4].

expansion of the charge-particle phase shift. For the corresponding leading-order MQIM (MQIM-LO) [4], the emerging particles are the charge $c$ and spin $s$ (or $s 1$ ) pseudofermions. For simplicity, in this paper we call them charge $c$ and spin $s$ particles, respectively. Both the general MQIM [3] and the MQIM-LO used in the studies of Ma et al. [4] provide accurate high-energy spectral function expressions beyond the low-energy TLL theory [2]. For our purposes, by high energy we mean energy scales beyond the TLL limit.

Except for accounting for higher-order terms in the effective-range expansion of the charge-particle phase shift, the expressions of the spectral-function quantities have for the higher-order MQIM (MQIM-HO) [45] the same general form as for the MQIM-LO. Within the MQIM-HO, the Hamiltonian that describes the 1D metallic states in the corresponding class of electronic lattice systems is of the form,

$$
\begin{aligned}
& \hat{H}=-t \sum_{\sigma=\uparrow, \downarrow} \sum_{j=1}^{L}\left(c_{j, \sigma}^{\dagger} c_{j+1, \sigma}+c_{j+1, \sigma}^{\dagger} c_{j, \sigma}\right)+\hat{V}_{R}, \\
& \hat{V}_{R}=\sum_{r=0}^{L / 2-1} V_{e}(r) \sum_{\sigma=\uparrow, \downarrow} \sum_{\sigma^{\prime}=\uparrow, \downarrow} \sum_{j=1}^{L} \hat{\rho}_{j, \sigma} \hat{\rho}_{j+r, \sigma^{\prime}},
\end{aligned}
$$

where $\hat{\rho}_{j, \sigma}=\left(c_{j, \sigma}^{\dagger} c_{j, \sigma}-\frac{1}{2}\right), V_{e}(0)=U / 2, V_{e}(r)=U F_{e}(r) / r$ for $r>0$, and $F_{e}(r)$ is a continuous decreasing screening function such that $F_{e}(0) \leqslant 1 / 4$, which at large $r$ vanishes as some inverse power of $r, \lim _{r \rightarrow \infty} F_{e}(r)=0$. The microscopic interactions associated with the electronic potentials then decay faster than $1 / r$. Hence the Fourier transform of $V_{e}(r)$ does not diverge at $k \rightarrow 0$ and the compressibility and sound velocity remain finite.

The matrix elements in the one-electron spectral function involve phase shifts and the charge parameter $\tilde{\xi}_{c}=$ $\sqrt{2 \tilde{K}_{c}}$ naturally related to them. Its range for the present lattice systems is $\left.\tilde{\xi}_{c}=\sqrt{2 \tilde{K}_{c}} \in\right] 1 / 2, \xi_{c}$ ]. Here $\tilde{K}_{c}$ is the usual TLL charge parameter and the bare charge parameters $\xi_{c} \in$ ]1, $\sqrt{2}$ [ and $K_{c}$ refer to the 1DHM in which the model Hamiltonian, Eqs. (9), becomes in the $\tilde{\xi}_{c} \rightarrow \xi_{c}$ limit. For electronic density $\left.n_{e} \in\right] 0,1\left[\right.$, there is a $\xi_{c} \rightarrow \tilde{\xi}_{c}$ transformation [4] for each fixed value of $\xi_{c}$ and $\tilde{\xi}_{c}$ that maps the 1DHM onto that model Hamiltonian, upon gently turning on $F_{e}(r)$. Consistent, $\lim _{\tilde{\xi}_{c} \rightarrow \xi_{c}} F_{e}(r) \rightarrow 0$ for $r \in[0, \infty]$. The MQIM-HO relies on that transformation. It transforms the 1DHM pseudofermion dynamical theory (PDT) [44], which for integrable models is equivalent to the MQIM [3,44], into the MQIM-HO that accounts for the electronic finite-range interactions of a class of electronic lattice systems whose 1D metallic states are described by the model Hamiltonian, Eqs. (9).

As reported by Ma et al. [4], the $\xi_{c} \rightarrow \tilde{\xi}_{c}$ transformation gives rise to a continuous decreasing of the initial bare parameters $\left.\xi_{c}=\sqrt{2 K_{c}} \in\right] 1, \sqrt{2}\left[\right.$ and $\left.K_{c}=\xi_{c}^{2} / 2 \in\right] 1 / 2,1[$. (Here $\xi_{c}=1$ for $u=U / 4 t \rightarrow \infty$ and $\xi_{c}=\sqrt{2}$ for $u \rightarrow 0$, respectively.) The resulting smaller renormalized parameter, $\tilde{\xi}_{c}=$ $\sqrt{2 \tilde{K}_{c}}$, has values in the ranges $\left.\tilde{\xi}_{c}=\sqrt{2 \tilde{K}_{c}} \in\right] 1 / 2,1[$ and $\left.\left.\tilde{\xi}_{c}=\sqrt{2 \tilde{K}_{c}} \in\right] 1, \xi_{c}\right]$. The theory does not apply at the bare parameter $\xi_{c}=1$ that refers to a nonmetallic Mott-Hubbard insulating phase at $n_{e}=1$ for $u>0$ and to $u \rightarrow \infty$ states whose spin configurations are all degenerated for $\left.n_{e} \in\right] 0,1[$. It also does not apply at $\tilde{\xi}_{c}=1$. Hence $\left.\tilde{K}_{c} \in\right] 1 / 8,1 / 2[$ and $\left.\tilde{K}_{c} \in\right] 1 / 2, K_{c}$ [, so that, as expected [42], $\tilde{K}_{c}>1 / 8$ for lattice correlated models.

Importantly, upon decreasing $\tilde{\xi}_{c}$ from $\tilde{\xi}_{c}=\xi_{c}$, the initial 1DHM SDS exponent $\left.\alpha_{0}=\left(2-\xi_{c}^{2}\right)^{2} /\left(8 \xi_{c}^{2}\right) \in\right] 0,1 / 8[$ continuously increases. Its expression is given by $\alpha=$ $\left(2-\tilde{\xi}_{c}^{2}\right)^{2} /\left(8 \tilde{\xi}_{c}^{2}\right)$. It has values in the corresponding intervals $\alpha \in\left[\alpha_{0}, 1 / 8[\right.$ and $\alpha \in] 1 / 8,49 / 32[$. The regime of more physical interest is $\left.\tilde{\xi}_{c} \in\right] 1 / 2,1[$, for which $\alpha>1 / 8$.

For each chosen initial fixed 1DHM finite values $u=$ $U / 4 t \in] 0, \infty\left[\right.$ and $\left.\xi_{c}=\xi_{c}\left(u, n_{e}\right) \in\right] 1, \sqrt{2}[$, where the electronic density varies in the interval $\left.n_{e} \in\right] 0,1[$ there is one $\xi_{c} \rightarrow \tilde{\xi}_{c}$ transformation. Indeed, the system retains the memory of $\xi_{c}$, and both $\xi_{c}$ and $\tilde{\xi}_{c}$ are MQIM-HO parameters that appear in the expressions for physical quantities. The same applies to the scattering lengths $a$ and $\tilde{a}$ considered below in Sec. IVB. The 1DHM initial interaction value $U$ remains under the $\xi_{c} \rightarrow \tilde{\xi}_{c}$ transformation, the interaction in both the on-site, $V_{e}(0)=U / 2$ and $r>0$, $V_{e}(r)=U F_{e}(r) / r$, parts of the electronic potential in Eqs. (9).

\section{B. The one-electron removal spectral function and its exponent's phase shifts}

Within the MQIM-HO, the one-electron removal spectral function in the $(k, \omega)$-plane vicinity of three singular features called spin $s$ branch line and charge $c$ and $c^{\prime}$ branch lines, 

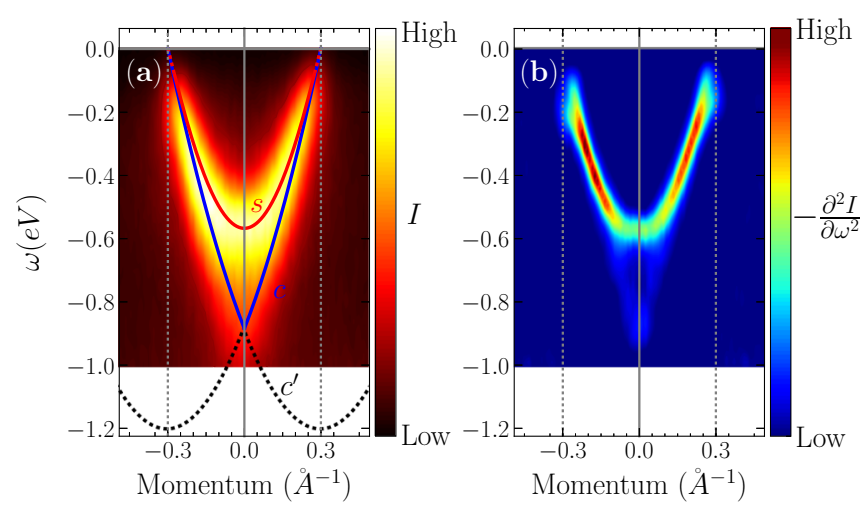

FIG. 6. (a) Raw ARPES data image of $\mathrm{MoSe}_{2}$ line defects with energy versus momentum $\left(\mathrm{k}_{\|}\right)$along the $\overline{\Gamma_{01}} \bar{K}$ direction in the Brillouin zone plus the theoretical $c, c^{\prime}$, and $s$ branch lines spectra [4] for $u=U / 4 t=0.18$, transfer integral $t=0.58 \mathrm{eV}$, and electronic density $n_{e}=2 / 3$. The full and dashed lines refer to momentum ranges with negative and positive exponents, respectively. (b) Second-derivative ARPES images. Source: The experimental ARPES data are from Ma et al. [4].

respectively, shown in Fig. 6(a), has the form

$$
\begin{aligned}
& \tilde{B}(k, \omega)=C_{s}\left(\tilde{\omega}_{s}(k)-\omega\right)^{\tilde{\xi}_{s}(k)} \text { and } \\
& \tilde{B}(k, \omega) \approx \sum_{\iota= \pm 1}(\iota) C_{\beta, l} \operatorname{Im}\left\{(-\iota)\left(\tilde{\omega}_{\beta}(k)-\omega-\frac{i}{2 \tau_{\beta}(k)}\right)^{\tilde{\tilde{S}}_{\beta}(k)}\right\},
\end{aligned}
$$

respectively, for small $\left(\tilde{\omega}_{s}(k)-\omega\right)>0$ and $\left(\tilde{\omega}_{\beta}(k)-\omega\right)>0$ where $\beta=c, c^{\prime}$. Here $C_{s}$ and $C_{\beta, l}$ are $n_{e}, u=U / 4 t$, and $\tilde{\xi}_{c^{-}}$ dependent constants and $\omega<0$ are high energies.

On the one hand, for $\tilde{\xi}_{c} \in\left[\tilde{\xi}_{c}^{\oslash}, \xi_{c}\right]$, the $\beta=c, c^{\prime}$ lifetimes $\tau_{\beta}(k)$ in Eqs. (10) are very large for the $k$ intervals for which the $\beta=c, c^{\prime}$ exponents $\tilde{\zeta}_{\beta}(k)$ are negative, so that the expression given in that equation is nearly power-lawlike, $\tilde{B}(k, \omega) \propto\left(\tilde{\omega}_{\beta}(k)-\omega\right)^{\tilde{\xi}_{\beta}(k)}$. The charge parameter value $\tilde{\xi}_{c}^{\ominus}=1 / \xi_{c}$ is determined by that of the bare charge parameter $\xi_{c}$ and varies in the interval $\tilde{\xi}_{c}^{\ominus} \in[1 / \sqrt{2}, 1$ [. Its smallest value $\tilde{\xi}_{c}^{\ominus}=1 / \sqrt{2}$ refers to $\xi_{c}=\sqrt{2}$ and $u \rightarrow 0$ whereas its nonreachable largest value $\tilde{\xi}_{c}^{\oslash} \rightarrow 1$ corresponds to $\xi_{c} \rightarrow 1$ for $u \rightarrow \infty$. On the other hand, the effects of long-range interactions are stronger for $\left.\tilde{\xi}_{c} \in\right] 1 / 2, \tilde{\xi}_{c}^{\oslash}$ ].

The $\gamma=s, c, c^{\prime}$ branch-line spectra $\tilde{\omega}_{\gamma}(k)$ in Eqs. (10) are provided in Eqs. (B1) of Appendix B. They involve the $c$ - and $s$-band-energy dispersions given in Eqs. (B2) of that Appendix. The excitation momentum $k$ in those spectra argument are in Eqs. (B1) of the same Appendix expressed in terms of the occupancies of the $c$-band momenta $q \in$ $\left[-2 k_{F}, 2 k_{F}\right]$ and $s$-band momenta $q^{\prime} \in\left[-k_{F}, k_{F}\right]$ associated with the corresponding excited states. Here $2 k_{F}=\pi n_{e}$.

Moreover, $\tau_{c}(k)$ and $\tau_{c^{\prime}}(k)$ are in Eqs. (10) large chargehole mobile impurity lifetimes mentioned above. They are associated with the relaxation processes discussed below and the expressions of the $\gamma=s, c, c^{\prime}$ exponents $\tilde{\zeta}_{\gamma}(k)$ in Eqs. (10) are given in Eqs. (B3) of Appendix B. They involve the charge parameter $\tilde{\xi}_{c}$ and the $c$ particle phase shifts $\tilde{\Phi}_{c, s}\left(\iota 2 k_{F}, q^{\prime}\right)$ and $\tilde{\Phi}_{c, c}\left(\iota 2 k_{F}, q\right)$ where $\iota= \pm 1$. They are the phase shifts in units

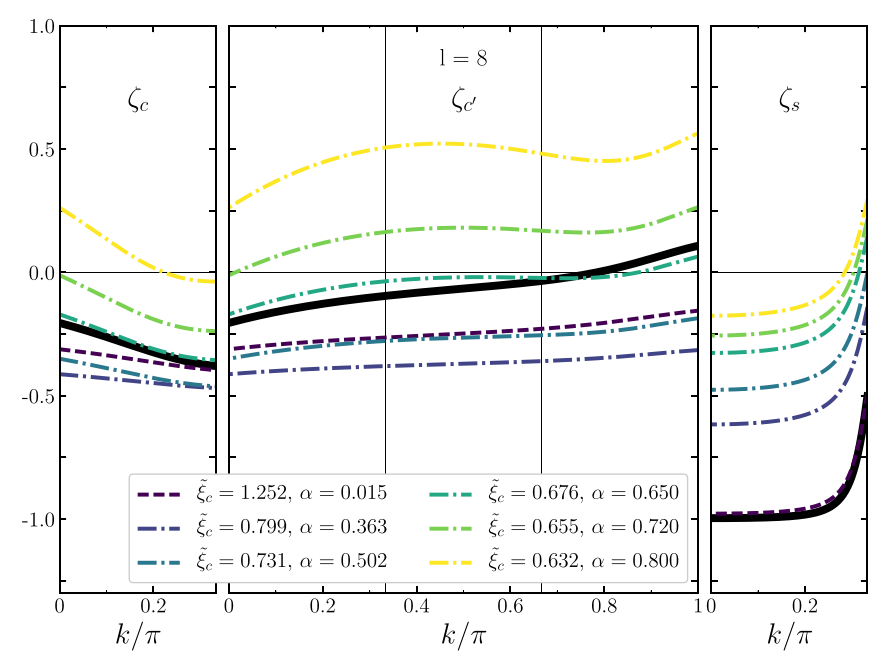

FIG. 7. The exponents that control the line shape near the $\mathrm{MoSe}_{2}$ line defect ARPES peaks and corresponding theoretical $c, c^{\prime}$, and $s$ branch lines, respectively, in Fig. 6(a). They are here plotted as a function of the momentum $k$ for $u=0.18, n_{e}=2 / 3, l=8$, and different $\tilde{\xi}_{c}$ and thus $\alpha$ values. The black solid lines refer to the conventional 1D Hubbard model $\left(\alpha_{0}=0.0011336\right.$ and $\left.\xi_{c}=1.367\right)$ and the red dashed and blue (dashed-dotted and full) lines to $\alpha<1 / 8$ and $\alpha>1 / 8$ values, respectively. The $c$ line, $c^{\prime}$ line, and $s$ line whose negative exponent ranges agree with the ARPES $(k, \omega)$-plane peaks in Fig. 6(a) are those whose $c^{\prime}$ branch-line exponent crosses zero at $k / \pi=0$. For such lines, $\tilde{\xi}_{c}=0.655, \alpha=0.72$, and $R_{\text {eff }}=1.01$ in units of lattice spacing. The $\tilde{\xi}_{c}$ value below which the effects of long-range interactions become stronger is $\tilde{\xi}_{c}^{\ominus}=1 / \xi_{c}=0.731$.

of $2 \pi$ imposed on a $c$ particle of momentum $\iota 2 k_{F}= \pm 2 k_{F}$ by a $s$ (spin) and $c$ (charge) hole mobile impurity created at momentum $q^{\prime}$ and $q$, respectively, under one-electron removal excitations. Such exponents expressions also involve phase shifts $\tilde{\Phi}_{s, s}\left( \pm k_{F}, q^{\prime}\right)$ and $\tilde{\Phi}_{s, c}\left( \pm k_{F}, q\right)$ imposed on the $s$ particles by a $s$ (spin) and $c$ (charge) hole mobile impurity, respectively. They remain hidden because they are invariant under the $\xi_{c} \rightarrow \tilde{\xi}_{c}$ transformation and due to the SU(2) symmetry are interaction, density, and momentum independent, as given in Eqs. (B5) of Appendix B. The exponents $\tilde{\zeta}_{\gamma}(k)$ are plotted in Fig. 7 as a function of the excitation momentum $k$ for $u=U / 4 t=0.18$ and electronic density $n_{e}=2 / 3$.

In the low-energy TLL regime and in the crossover regime to it that refer to small-energy regions near the $(k, \omega)$-plane points $\left( \pm k_{F}, \omega\right)$ for the $s$ and $c$ branch lines and $\left( \pm 3 k_{F}, \omega\right)$ for the $c^{\prime}$ branch line, the corresponding exponent expressions are different from those provided in Eqs. (B3) of Appendix B. Fortunately, the ARPES peaks studied here refer to higher energy scales at which the latter exponents apply.

The microscopic processes that control the weight distribution near the $\gamma=s, c, c^{\prime}$ branch-line singularities of the one-electron removal spectral function at $k$ domains for which the exponents $\tilde{\zeta}_{\gamma}(k)$ in Eqs. (B3) of Appendix B are negative refer to creation of one hole in the $c$ band and one hole in the $s$ band. Specifically, in the case of the $s$ branch line, the $s$-band hole is created away from the corresponding Fermi points $\pm k_{F}$ whereas the $c$-band hole is created at one of that band Fermi points $\pm 2 k_{F}$. The charge $c$ and $c^{\prime}$ branch lines result from processes under which the $c$-band hole is created 
away from the corresponding Fermi points $\pm 2 k_{F}$ and the $s$-band hole is created at one of its bands, Fermi points $\pm k_{F}$. Furthermore, the $c$-band discrete momenta are all shifted by $\pi / L$ or $-\pi / L$ whereas those of the $s$ band are not. This leads to an overall macroscopic shift of momentum $2 k_{F}$ or $-2 k_{F}$, respectively, which originates from the shifting of the whole $c$-band occupied sea.

Such a shifting is behind the existence of two independent charge branch lines. The parts of these two branch lines that connect the point $(k, \omega)=\left(-k_{F}, 0\right)$ in Fig. 6(a) to a $k=0$ finite- $\omega$ point and the latter point to $(k, \omega)=\left(k_{F}, 0\right)$ are here and in the figure called the $c$ branch line. The remaining parts of the charge branch lines that connect the point at $k=0$ and finite $\omega$ to the $(k, \omega)=\left(-3 k_{F}, 0\right)$ and $(k, \omega)=\left(3 k_{F}, 0\right)$ points, respectively, are called the $c^{\prime}$ branch line. (Because one finds below that for the parameters suitable to the theoretical description of the ARPES in the $\mathrm{MoSe}_{2}$ line defects, there are no singularities in the $c^{\prime}$ branch line, in Fig. 6(a) only part of its $k$ range is included.)

Only the charge hole or spin hole, respectively, that is created away from the corresponding Fermi points is called a mobile impurity. The high-energy MQIM-HO charge-hole quantum mobile impurity and spin-hole quantum mobile impurity become in the low-energy limit the usual TLL holon and spinon, respectively.

On the one hand, since the $c$ and $c^{\prime}$ branch lines lie in the spectral-weight continuum, in their vicinity the spectralfunction expression given in Eqs. (10) is for the charge parameter range $\left.\tilde{\xi} \in] 1 / 2, \tilde{\xi}_{c}^{\oslash}\right]$ for which the effects of the finite-range interactions are stronger such that their power-law singularities are slightly broadened by weak charge-hole mobile impurity relaxation effects associated with large lifetimes $\tau_{c}(k)$ and $\tau_{c^{\prime}}(k)$. However, they remain sharp peaks for the $k$ ranges for which the exponents $\tilde{\zeta}_{c}(k)$ and $\tilde{\zeta}_{c^{\prime}}(k)$, respectively, given in Eqs. (B3) of Appendix B are negative. For $\tilde{\xi}>\tilde{\xi}_{c}^{\oslash}=$ $1 / \xi_{c}$, the relaxation effects are much weaker and the above reported $\beta=c, c^{\prime}$ branch line singularities' power-law behavior $\tilde{B}(k, \omega) \propto\left(\tilde{\omega}_{\beta}(k)-\omega\right)^{\tilde{\xi}_{\beta}(k)}$ is a good approximation for their expression given in Eqs. (10). What matters for the description of the $\mathrm{MoSe}_{2}$ line defect ARPES peaks distribution reported below in Sec. IVC is not, though, the precise form of the theoretical spectral function near its peaks but rather the $k$ ranges for which its exponents are negative. They provide precise and valuable information on the predicted location of such peaks in the $(k, \omega)$ plane.

On the other hand, the $s$ branch line coincides with an edge of support of the spectral function that limits the finite-weight region. Then the scattering processes allowed by energy and momentum conservation ensure that the expression of the exponent $\tilde{\zeta}_{s}(k)$ in Eqs. (10) is exact.

The $s$ particle energy dispersion remains invariant under the $\xi_{c} \rightarrow \tilde{\xi}_{c}$ transformation. The $c$-particle energy dispersion bandwidth of the occupied sea increases slightly [45]. (See Eqs. (B2) of Appendix B where $\tilde{\varepsilon}_{c}(q)$ and $\tilde{\varepsilon}_{s}\left(q^{\prime}\right)=\varepsilon_{s}\left(q^{\prime}\right)$ are the MQIM-HO energy dispersions and $\varepsilon_{c}(q)$ and $\varepsilon_{s}\left(q^{\prime}\right)$ are those associated with the bare limit, $\tilde{\xi}_{c}=\xi_{c}$, that refers to the 1DHM.) That the spin spectra remain invariant under finite-range interactions whereas the charge spectra bandwidth and charge Fermi velocity are increased upon increasing the interactions range is also known from numerical studies
[46]. (See charge and spin spectra in Fig. 7 of that paper and corresponding discussion.)

However, the major effects of the finite-range interactions are on the one-electron matrix elements between the ground state and the excited states. In the representation in terms of charge and spin particles, such effects lead to a renormalization of the phase shifts of the charge particles imposed by the charge- and spin-hole mobile impurities created under transitions to the one-electron removal excited states [4]. The renormalization of the phase shifts $2 \pi \tilde{\Phi}_{c, s}\left( \pm 2 k_{F}, q^{\prime}\right)$ and $2 \pi \tilde{\Phi}_{c, c}\left( \pm 2 k_{F}, q\right)$ appearing in the exponents expressions, Eqs. (B3) of Appendix B, under the $\xi_{c} \rightarrow \tilde{\xi}_{c}$ transformation leads to Eqs. (B4) of Appendix B.

The MQIM-HO phase shift term $2 \pi \tilde{\Phi}_{c, c}^{R_{\text {eff }}}\left(k_{r}\right)$ in that equation is absent from the 1DHM as it emerges from finiterange interactions higher-order effects beyond the renormalization factor $\left[\xi_{c}\left(\tilde{\xi}_{c}-1\right)^{2}\right] /\left[\tilde{\xi}_{c}\left(\xi_{c}-1\right)^{2}\right]$ of the phase-shift term $2 \pi \tilde{\Phi}_{c, c}^{\tilde{a}}\left( \pm 2 k_{F}, q\right)$. (That term has not been considered in the MQIM-LO of Ma et al. [4].)

Such higher-order effects result from the potential $V_{c}(x)$ associated with the interaction of the charge $c$ particle and the charge-hole mobile impurity at spatial distance $x$, which is induced by the electronic potential $V_{e}(r)$ in Eqs. (9). For the class of MQIM-HO electronic potentials, that induced potential $V_{c}(x)$ vanishes for large $x$ as $V_{c}(x)=-C_{c} / x^{l}$. Here $l \geqslant 6$ is an integer determined by the large- $r$ behavior of $V_{e}(r), C_{c}=\left(2 r_{l}\right)^{l-2} / \mu, r_{l}$ is a length scale (van der Waals length for $l=6$ ), and $\mu$ is the reduced mass [45].

The phase-shift term $2 \pi \tilde{\Phi}_{c, c}^{R_{\text {eff }}}\left(k_{r}\right)$ expression [45] involves the effective range $R_{\text {eff }}$ of the interactions between the $c$ particles at and near the $c$-band Fermi points $\pm 2 k_{F}$ and the charge-hole mobile impurity created under one-electron removal excitations at $c$-band momenta $q$ away from the $c$-band Fermi points. It is a function of the corresponding relative momentum, $k_{r}=q \mp 2 k_{F}$, such that $\left|k_{r}\right| \in\left[k_{F c}^{0}, 4 k_{F}\right.$. The use of standard scattering theory for potentials with large- $x$ behavior $V_{c}(x)=-C_{c} / x^{l}$ where $l \geqslant 6$ leads to a $R_{\text {eff }}$ effective range expression that involves the ratio $\tilde{a} / a$ of the scattering length $\tilde{a}$ corresponding to the renormalized charge parameter $\tilde{\xi}_{c}$ value and the bare scattering length $a$ associated with the $n_{e}$ and $u=U / 4 t$ dependent bare charge parameter $\xi_{c}$ value, respectively [45].

\section{Application to the ARPES peaks distribution}

The higher-order charge-charge interaction effects associated with the phase-shift term $2 \pi \tilde{\Phi}_{c, c}^{R_{\text {eff }}}\left(k_{r}\right)$ play an important role in the one-electron spectral properties of $1 \mathrm{D}$ metallic states as those in a bismuth-induced anisotropic structure on indium antimonide [Bi/InSb(001)] whose effective range $R_{\text {eff }}$ can reach values $R_{\text {eff }} \approx 17$ in units of lattice spacing [45].

The studies of Ma et al. [4] on the $\mathrm{MoSe}_{2}$ line defects considered that $R_{\text {eff }}=0$ and thus that $2 \pi \tilde{\Phi}_{c, c}^{R_{\text {eff }}}\left(k_{r}\right)=0$ in the expression of the phase shift $2 \pi \tilde{\Phi}_{c, c}\left( \pm 2 k_{F}, q\right)$ in Eqs. (B4). This is acceptable provided that $R_{\text {eff }} \approx 1$ in units of lattice spacing. Here we confirm that such a condition holds for the $\mathrm{MoSe}_{2}$ line defects. Nevertheless, we show that accounting for the effects of $R_{\text {eff }}$ improves the agreement with the experiments beyond that reached by Ma et al. [4]. 
As in that reference, the SDS exponent $\alpha=(2-$ $\left.\tilde{\xi}_{c}^{2}\right)^{2} /\left(8 \tilde{\xi}_{c}^{2}\right)$ is chosen to refer to the $\tilde{\xi}_{c}$ value for which there is agreement between the specific $k$ intervals at which the $\gamma=s, c, c^{\prime}$ branch-lines exponents $\tilde{\zeta}_{\gamma}(k)$ given in Eqs. (B3) of Appendix B are negative and the ARPES peaks distribution. For the $c$ and $s$ branch lines, these intervals are $k \in\left[-2 k_{F}+k_{F c}^{\mathrm{ex}}, 2 k_{F}-k_{F c}^{\mathrm{ex}}\right]$ and $k \in\left[-k_{F}+k_{F s}^{\mathrm{ex}}, k_{F}-k_{F s}^{\mathrm{ex}}\right]$, respectively. On the one hand, here $k_{F c}^{\mathrm{ex}}$ is the experimental momentum that corresponds to the theoretical small momentum $k_{F c}^{0}$ that controls the TLL and cross over to TLL regimes momentum width considered in the discussions of Sec. IV B. Consistent with those discussions, $k_{F c}^{\mathrm{ex}} / k_{F}$ is vanishing or very small. On the other hand, $k_{F s}^{\mathrm{ex}}>k_{F s}^{0}$ such that $k_{F s}^{\mathrm{ex}} / k_{F} \approx 0.12$ rather refers to the experimental momenta $k= \pm\left(k_{F}-k_{F s}^{\text {ex }}\right)$ at which the theoretical $s$-branch exponent vanishes. Hence it is negative and positive for $k \in\left[-k_{F}+k_{F s}^{\mathrm{ex}}, k_{F}-k_{F s}^{\mathrm{ex}}\right]$ and $k \in$ $\left[-k_{F}+k_{F s}^{\mathrm{ex}},-k_{F}\right] ;\left[k_{F}-k_{F s}^{\mathrm{ex}}, k_{F}\right]$, respectively. Indeed, only for negative exponent values does the theoretical $s$ branch line correspond to ARPES peaks. (See $s$ branch line exponent in Fig. 7 for the value $\alpha=0.72$, for which, as discussed below, there is agreement between theory and experiments.) Finally, the $c^{\prime}$ branch line exponent should be positive for its whole $k$ interval.

The exponents in Eqs. (B3) of Appendix B depend both on $\tilde{\xi}_{c}$ and momentum-dependent phase shifts $\tilde{\Phi}_{c, c}\left( \pm 2 k_{F}, q\right)$ and $\tilde{\Phi}_{c, s}\left( \pm 2 k_{F}, q^{\prime}\right)$. There is no apparent direct relation between the high-energy ARPES peaks distribution and the low-energy SDS. That the MQIM-HO contains the main microscopic mechanisms behind the 1D metallic states physics in the $\mathrm{MoSe}_{2}$ line defects then requires that the $\alpha$ value that refers to the $\tilde{\xi}_{c}$ value for which there is agreement with the high-energy ARPES peaks distribution is also that measured within the low-energy angle integrated photoemission intensity.

We use in the expressions of the exponents $\tilde{\zeta}_{c}(k)$ and $\tilde{\zeta}_{c^{\prime}}(k)$, Eqs. (B3) of Appendix B, the expression of the phase shift $2 \pi \widetilde{\Phi}_{c, c}\left( \pm 2 k_{F}, q\right)$ in Eqs. (B4) of that Appendix, which includes the term $\tilde{\Phi}_{c, c}^{R_{\text {eff }}}\left(k_{r}\right)$. We then find that the parameter values that at electronic density $n_{e}=2 / 3$ lead to agreement between the above intervals of the $s, c$, and $c^{\prime}$ branch lines [see Fig. 6(a)] and the line defect ARPES peaks distribution are $u=U / 4 t=0.18, \tilde{\xi}_{c}=0.655, \alpha=0.72$, and $l=8$ for transfer integral $t=0.58 \mathrm{eV}$.

The corresponding $\gamma=c, c^{\prime}, s$ exponents $\tilde{\zeta}_{\gamma}(k)$ are plotted as a function of $k$ in Fig. 7 for different $\tilde{\xi}_{c}$ values and corresponding $\alpha=\left(2-\tilde{\xi}_{c}^{2}\right)^{2} /\left(8 \tilde{\xi}_{c}^{2}\right)$ values. The $\tilde{\xi}_{c}$ value below which the effects of long-range interactions become stronger is $\tilde{\xi}_{c}^{\oslash}=1 / \xi_{c}=0.731$. The matching $\alpha=0.72$ value refers to $\tilde{\xi}_{c}=0.655$ and $R_{\text {eff }}=1.01$ in units of lattice spacing and agrees with the estimated experimental uncertainty, $\alpha=$ $0.75 \pm 0.05$ [4]. The prediction of Ma et al. [4] that $\alpha=$ 0.78 lays in that uncertainty range, which confirms that the approximation of using $R_{\text {eff }} \approx 0$ in the expression of the phase shift $2 \pi \tilde{\Phi}_{c, c}\left( \pm 2 k_{F}, q\right)$ is acceptable.

The room-temperature experimental SDS of the $\mathrm{MoSe}_{2}$ line defects is plotted in Fig. 5 along with analytical lines for $\alpha=0.70,75,80$. The theoretical SDS universal power-law behavior controlled by the exponent $\alpha$ in Fig. 5, though, only applies at very low energy, up to $\approx 0.07 \mathrm{eV}$. For larger energy values, the SDS loses its universal power-law behavior, its form becoming different and specific to each many-electron problem.

Comparison with the experimental points for that energy range reveals that concerning the $\alpha=0.70,75,80$ theoretical lines, the best agreement is reached at $\alpha=0.70$. This is consistent with our correction from $\alpha=0.78$ to $\alpha=0.72$, improving the agreement. This is physically appealing, as one expects that the effective range should not be smaller than one lattice spacing.

\section{CONCLUSIONS}

Confined states at TGBs in $\mathrm{MoSe}_{2}$ were shown to be well described by a three-orbital TB model, which is robust to the details of the defect hoppings. The presence of a single band (per spin) at the Fermi level is consistent with experiments.

Modeling the confined states as a 1D interacting electronic system unveils a MQIM $(k, \omega)$-plane behavior with an effective range for the charge fractionalized particle-charge-hole mobile impurity interaction that extends up to the lattice spacing, in excellent agreement with ARPES measurements.

The robustness and the properties found here for 1D confined states in $\mathrm{MoSe}_{2}$ extend to the full semiconducting TMD family, giving rise to a distinct paradigm where onedimensionality is protected by the two-dimensionality of the host material.

\section{ACKNOWLEDGMENTS}

T.Č. and J.M.P.C. thank Hai-Qing Lin for interesting discussions. E.C. is grateful to Pedro Ribeiro for valuable insights regarding the absence of a bound state in the low energy description. J.M.P.C. thanks Maria C. Asensio, Matthias Batzill, and Francisco Guinea for illuminating discussions, Boston University's Condensed Matter Theory Visitors Program for support, and the hospitality of MIT. We acknowledge the support from NSAF U1530401 and computational resources from CSRC (Beijing), the Portuguese FCT through Grants No. UID/FIS/04650/2013, No. UID/CTM/04540/2013, No. PTDC/FIS-MAC/29291/ 2017, and No. SFRH/BSAB/142925/2018, and NSFC Grant No. 11650110443.

T.C. and L.L. contributed equally to this work.

\section{APPENDIX A: DERIVATION OF THE CONTINUUM THEORY}

Consider the three-band TB Hamiltonian of TMDs [16] applied to the $y<0$ side of the TGB [see Fig. 1(a)],

$$
\mathcal{H}=\sum_{\mathbf{k}} \hat{\psi}_{\mathbf{k}}^{\dagger} H(\mathbf{k}) \hat{\psi}_{\mathbf{k}},
$$

with $\hat{\psi}_{\boldsymbol{k}}^{\dagger}=\left(\hat{c}_{\boldsymbol{k}, z^{2}}^{\dagger}, \hat{c}_{\boldsymbol{k}, x y}^{\dagger}, \hat{c}_{\boldsymbol{k}, x^{2}-y^{2}}^{\dagger}\right)$ and

$$
H(\mathbf{k})=\left(\begin{array}{ccc}
h_{0} & h_{1} & h_{2} \\
h_{1}^{*} & h_{11} & h_{12} \\
h_{2}^{*} & h_{12}^{*} & h_{22}
\end{array}\right),
$$


where

$$
\begin{aligned}
h_{0} & =\epsilon_{1}+2 t_{0} \cos 2 \alpha+4 t_{0} \cos \alpha \cos \beta, \quad h_{11}=\epsilon_{2}+2 t_{11} \cos 2 \alpha+\left(t_{11}+3 t_{22}\right) \cos \alpha \cos \beta, \\
h_{22} & =\epsilon_{2}+2 t_{22} \cos 2 \alpha+\left(t_{22}+3 t_{11}\right) \cos \alpha \cos \beta, \quad h_{1}=2 i t_{1} \sin 2 \alpha+2 i t_{1} \sin \alpha \cos \beta-2 \sqrt{3} t_{2} \sin \alpha \sin \beta, \\
h_{2} & =2 t_{2} \cos 2 \alpha-2 t_{2} \cos \alpha \cos \beta+2 \sqrt{3} i t_{1} \cos \alpha \sin \beta, \quad h_{12}=2 i t_{12} \sin 2 \alpha-4 i t_{12} \sin \alpha \cos \beta+\sqrt{3}\left(t_{22}-t_{11}\right) \sin \alpha \sin \beta,
\end{aligned}
$$

$\alpha=k_{x} a / 2$, and $\beta=\sqrt{3} k_{y} a / 2$. The $K(\tau=+1)$ and $K^{\prime}(\tau=-1)$ points in the BZ are

$$
\tau \mathbf{K}=\left(\tau \frac{4 \pi}{3 a}, 0\right)
$$

where $\alpha$ and $\beta$ take the values $\alpha_{\tau}=\tau \frac{2 \pi}{3}, \beta_{\tau}=0$. The Taylor expansion to the second order around $K$ and $K^{\prime}$ points reads

$$
\begin{aligned}
H(\tau \mathbf{K}+\mathbf{q}) & =H(\tau \mathbf{K})+H_{\mathbf{q}}^{(1)}+H_{\mathbf{q}}^{(2)}+\mathcal{O}(a q)^{3} \\
& =H(\tau \mathbf{K})+q_{i}\left(\partial_{i} H\right)_{\tau \mathbf{K}}+\frac{1}{2} q_{i} q_{j}\left(\partial_{i} \partial_{j} H\right)_{\tau \mathbf{K}}+\mathcal{O}(a q)^{3} \\
& =\left(\begin{array}{ccc}
\eta_{0} & \eta_{1} & \eta_{2} \\
\eta_{1}^{*} & \eta_{11} & \eta_{12} \\
\eta_{2}^{*} & \eta_{12}^{*} & \eta_{22}
\end{array}\right)+a\left(\begin{array}{ccc}
u_{0} & u_{1} & u_{2} \\
u_{1}^{*} & u_{11} & u_{12} \\
u_{2}^{*} & u_{12}^{*} & u_{22}
\end{array}\right)+a^{2}\left(\begin{array}{ccc}
v_{0} & v_{1} & v_{2} \\
v_{1}^{*} & v_{11} & v_{12} \\
v_{2}^{*} & v_{12}^{*} & v_{22}
\end{array}\right)+\mathcal{O}(a q)^{3},
\end{aligned}
$$

with

$$
\begin{aligned}
\eta_{0}=\epsilon_{1}-3 t_{0}, \quad \eta_{11}=\epsilon_{2}-\frac{1}{2}\left(3 t_{11}+3 t_{22}\right), \quad \eta_{22}=\epsilon_{2}-\frac{1}{2}\left(3 t_{11}+3 t_{22}\right), \quad \eta_{1}=0, \quad \eta_{2}=0, \quad \eta_{12}=-i \tau 3 \sqrt{3} t_{12}, \\
u_{0}=0, \quad u_{11}=\frac{3 \sqrt{3}}{4} \tau\left(t_{11}-t_{22}\right) q_{x}, \quad u_{22}=\frac{3 \sqrt{3}}{4} \tau\left(t_{22}-t_{11}\right) q_{x}, \quad u_{1}=-\frac{3}{2} i t_{1} q_{x}-\tau \frac{3 \sqrt{3}}{2} t_{2} q_{y}, \\
u_{2}=\tau \frac{3 \sqrt{3}}{2} t_{2} q_{x}-\frac{3}{2} i t_{1} q_{y}, \quad u_{12}=\tau \frac{3 \sqrt{3}}{4}\left(t_{22}-t_{11}\right) q_{y},
\end{aligned}
$$

and

$$
\begin{aligned}
& v_{0}=\frac{3}{4} t_{0} q^{2}, \quad v_{11}=\frac{3}{16}\left[\left(3 t_{11}+t_{22}\right) q_{x}^{2}+\left(t_{11}+3 t_{22}\right) q_{y}^{2}\right], \quad v_{22}=\frac{3}{16}\left[\left(t_{11}+3 t_{22}\right) q_{x}^{2}+\left(3 t_{11}+t_{22}\right) q_{y}^{2}\right], \\
& v_{1}=\frac{3}{4} t_{2} q_{x} q_{y}+i \tau \frac{3 \sqrt{3}}{8} t_{1}\left(q_{x}^{2}-q_{y}^{2}\right), \quad v_{2}=\frac{3}{8} t_{2}\left(q_{x}^{2}-q_{y}^{2}\right)-i \tau \frac{3 \sqrt{3}}{4} t_{1} q_{x} q_{y}, \quad v_{12}=\frac{3}{8}\left(t_{11}-t_{22}\right) q_{x} q_{y}+i \tau \frac{3 \sqrt{3}}{4} t_{12} q^{2} .
\end{aligned}
$$

Diagonalizing the zeroth-order Hamiltonian in Eq. (A5),

$$
H(\tau \mathbf{K})=\left(\begin{array}{ccc}
\epsilon_{1}-3 t_{0} & 0 & 0 \\
0 & \epsilon_{2}-\frac{1}{2}\left(3 t_{11}+3 t_{22}\right) & -i \tau 3 \sqrt{3} t_{12} \\
0 & i \tau 3 \sqrt{3} t_{12} & \epsilon_{2}-\frac{1}{2}\left(3 t_{11}+3 t_{22}\right)
\end{array}\right),
$$

one obtains for the respective eigenvectors and eigenvalues,

$$
\begin{aligned}
\left|\psi_{c}(\tau \mathbf{K})\right\rangle & =\left|\tau \mathbf{K}, d_{z^{2}}\right\rangle, \quad \epsilon_{c}=\epsilon_{1}-3 t_{0}, \quad\left|\psi_{v}(\tau \mathbf{K})\right\rangle=\frac{1}{\sqrt{2}}\left[\left|\tau \mathbf{K}, d_{x^{2}-y^{2}}\right\rangle+i \tau\left|\tau \mathbf{K}, d_{x y}\right\rangle\right], \\
\epsilon_{v} & =\epsilon_{2}-\frac{1}{2}\left(3 t_{11}+3 t_{22}\right)-3 \sqrt{3} t_{12}, \quad\left|\psi_{h}(\tau \mathbf{K})\right\rangle=\frac{1}{\sqrt{2}}\left[\left|\tau \mathbf{K}, d_{x^{2}-y^{2}}\right\rangle-i \tau\left|\tau \mathbf{K}, d_{x y}\right\rangle\right], \\
\epsilon_{h} & =\epsilon_{2}-\frac{1}{2}\left(3 t_{11}+3 t_{22}\right)+3 \sqrt{3} t_{12},
\end{aligned}
$$

with the underscripts meaning conduction band $(c)$, valence band $(v)$, and highest energy band $(h)$.

The transformation matrix that diagonalizes $H(\tau \mathbf{K})$ reads

$$
U_{\tau}=\left(\begin{array}{ccc}
1 & 0 & 0 \\
0 & -i \tau / \sqrt{2} & 1 \tau / \sqrt{2} \\
0 & i \tau / \sqrt{2} & 1 \tau / \sqrt{2}
\end{array}\right)
$$


and the first-order matrix in the eigenbasis of $H(\tau \mathbf{K})$ is to be written as

$$
\begin{aligned}
\Sigma^{(1)}(\mathbf{q}) & =U_{\tau} H^{(1)}(\mathbf{q}) U_{\tau}^{-1} \\
& =\left(\begin{array}{ccc}
u_{0} & \frac{1}{\sqrt{2}}\left(u_{2}+i \tau u_{1}\right) & \frac{1}{\sqrt{2}}\left(u_{2}-i \tau u_{1}\right) \\
\frac{1}{\sqrt{2}}\left(u_{2}+i \tau u_{1}\right)^{*} & \frac{1}{2}\left(u_{22}+u_{11}\right)+\tau \operatorname{Im}\left[u_{12}\right] & \frac{1}{2}\left(u_{22}-u_{11}\right)-i \tau \operatorname{Re}\left[u_{12}\right] \\
\frac{1}{\sqrt{2}}\left(u_{2}-i \tau u_{1}\right)^{*} & \frac{1}{2}\left(u_{22}-u_{11}\right)+i \tau \operatorname{Re}\left[u_{12}\right] & \frac{1}{2}\left(u_{22}+u_{11}\right)-\tau \operatorname{Im}\left[u_{12}\right]
\end{array}\right) \\
& =a\left(\begin{array}{ccc}
0 & t_{v c}^{(1)}\left(\tau q_{x}-i q_{y}\right) & t_{c h}^{(1)}\left(\tau q_{x}+i q_{y}\right) \\
t_{v c}^{(1)}\left(\tau q_{x}+i q_{y}\right) & 0 & t_{v h}^{(1)}\left(\tau q_{x}-i q_{y}\right) \\
t_{c h}^{(1)}\left(\tau q_{x}-i q_{y}\right) & t_{v h}^{(1)}\left(\tau q_{x}+i q_{y}\right) & 0
\end{array}\right),
\end{aligned}
$$

with

$$
t_{v c}^{(1)}=\frac{3}{2 \sqrt{2}}\left(\sqrt{3} t_{2}+t_{1}\right), \quad t_{c h}^{(1)}=\frac{3}{2 \sqrt{2}}\left(\sqrt{3} t_{2}-t_{1}\right), \quad t_{v h}^{(1)}=\frac{3 \sqrt{3}}{4}\left(t_{22}-t_{11}\right) .
$$

The second-order correction to the Hamiltonian can be written as

$$
\begin{aligned}
\Sigma^{(2)}(\mathbf{q}) & =U_{\tau} H^{(2)}(\mathbf{q}) U_{\tau}^{-1} \\
& =a^{2}\left(\begin{array}{ccc}
v_{0} & \frac{1}{\sqrt{2}}\left(v_{2}+i \tau v_{1}\right) & \frac{1}{\sqrt{2}}\left(v_{2}-i \tau v_{1}\right) \\
\frac{1}{\sqrt{2}}\left(v_{2}+i \tau v_{1}\right)^{*} & \frac{1}{2}\left(v_{22}+v_{11}\right)+\tau \operatorname{Im} v_{12} & \frac{1}{2}\left(v_{22}-v_{11}\right)-i \tau \operatorname{Re} v_{12} \\
\frac{1}{\sqrt{2}}\left(v_{2}-i \tau v_{1}\right)^{*} & \frac{1}{2}\left(v_{22}-v_{11}\right)+i \tau \operatorname{Re} v_{12} & \frac{1}{2}\left(v_{22}+v_{11}\right)-\tau \operatorname{Im} v_{12}
\end{array}\right) \\
& =a^{2}\left(\begin{array}{ccc}
\chi_{c} q^{2} & t_{v c}^{(2)}\left(q_{x}+i \tau q_{y}\right)^{2} & t_{c h}^{(2)}\left(q_{x}-i \tau q_{y}\right)^{2} \\
t_{v c}^{(2)}\left(q_{x}-i \tau q_{y}\right)^{2} & \chi_{v} q^{2} & t_{v h}^{(2)}\left(q_{x}+i \tau q_{y}\right)^{2} \\
t_{c h}^{(2)}\left(q_{x}+i \tau q_{y}\right)^{2} & t_{v h}^{(2)}\left(q_{x}-i \tau q_{y}\right)^{2} & \chi_{h} q^{2}
\end{array}\right),
\end{aligned}
$$

with

$$
\begin{aligned}
\chi_{c} & =\frac{3}{4} t_{0}, \quad \chi_{v}=\frac{3}{8}\left(t_{11}+t_{22}+\sqrt{3} t_{12}\right), \quad \chi_{h}=\frac{3}{8}\left(t_{11}+t_{22}-\sqrt{3} t_{12}\right), \\
t_{v c}^{(2)} & =\frac{3}{8 \sqrt{2}}\left(t_{2}-\sqrt{3} t_{1}\right), \quad t_{c h}^{(2)}=\frac{3}{8 \sqrt{2}}\left(t_{2}-\sqrt{3} t_{1}\right), \quad t_{v h}^{(2)}=\frac{3}{16}\left(t_{22}-t_{11}\right) .
\end{aligned}
$$

The effective second-order Hamiltonian of the lowest conduction and highest valence bands is then given by

$$
\begin{aligned}
H_{\mathrm{eff}}(\mathbf{q})= & P_{l}\left[H_{0}+\Sigma^{(1)}(\mathbf{q})+\Sigma^{(2)}(\mathbf{q})\right] P_{l}+\sum_{l=c, v} \frac{\left|\psi_{l}\right\rangle\left\langle\psi_{l}\left|\Sigma^{(1)}(\mathbf{q}) P_{h} \Sigma^{(1)}(\mathbf{q})\right| \psi_{l}\right\rangle\left\langle\psi_{l}\right|}{\epsilon_{l}-\epsilon_{h}} \\
& +\sum_{\substack{m, n=c, v \\
m \neq n}} \frac{\left|\psi_{m}\right\rangle\left\langle\psi_{m}\left|\Sigma^{(1)}(\mathbf{q}) P_{h} \Sigma^{(1)}(\mathbf{q})\right| \psi_{n}\right\rangle\left\langle\psi_{n}\right|}{\epsilon_{F}-\epsilon_{h}},
\end{aligned}
$$

with $P_{l}=\left|\psi_{c}\right\rangle\left\langle\psi_{c}|+| \psi_{v}\right\rangle\left\langle\psi_{v}\left|, P_{h}=\right| \psi_{h}\right\rangle\left\langle\psi_{h}\right|$, and $\epsilon_{F}=\frac{\epsilon_{c}+\epsilon_{v}}{2}$. After straightforward manipulation, we obtain

$$
\begin{aligned}
H_{\mathrm{eff}}^{(y<0)}(\mathbf{q})= & \left(\begin{array}{cc}
\epsilon_{c} & 0 \\
0 & \epsilon_{v}
\end{array}\right)+a t_{v c}^{(1)}\left(\begin{array}{cc}
0 & \tau q_{x}-i q_{y} \\
\tau q_{x}+i q_{y} & 0
\end{array}\right)+a^{2}\left(\begin{array}{cc}
\chi_{c} q^{2} & t_{v c}^{(2)}\left(q_{x}+i \tau q_{y}\right)^{2} \\
t_{v c}^{(2)}\left(q_{x}-i \tau q_{y}\right)^{2} & \chi_{v} q^{2}
\end{array}\right) \\
& +a^{2}\left(\begin{array}{cc}
\xi_{c} q^{2} & t_{v c h}\left(q_{x}+i \tau q_{y}\right)^{2} \\
t_{v c h}\left(q_{x}-i \tau q_{y}\right)^{2} & \xi_{v} q^{2}
\end{array}\right) \\
= & v \hbar\left(q_{x} \tau_{3} \sigma_{x}+q_{y} \sigma_{y}\right)+\left(\Delta+\delta \xi a^{2} q^{2}\right) \sigma_{3}+\zeta a^{2}\left(q_{x} \tau_{3} \sigma_{x}-q_{y} \sigma_{y}\right) \sigma_{x}\left(q_{x} \tau_{3} \sigma_{x}-q_{y} \sigma_{y}\right)+\left(\epsilon_{F}+\xi q^{2}\right) \sigma_{0},
\end{aligned}
$$

where $\xi_{c}=\frac{t_{c h}^{2}}{\epsilon_{c}-\epsilon_{h}}, \xi_{v}=\frac{t_{v h}^{2}}{\epsilon_{v}-\epsilon_{h}}, t_{v c h}=\frac{t_{v h} t_{c h}}{\epsilon_{-} \epsilon_{h}}, v=a t_{v c}^{(1)}, \Delta=\frac{\epsilon_{c}-\epsilon_{v}}{2}, \delta \xi=\frac{\xi_{c}-\xi_{v}}{2}+\frac{\chi_{c}-\chi_{v}}{2}, \zeta=t_{v c}^{(2)}+t_{v c h}, \xi=\frac{\chi_{c}+\chi_{v}+\xi_{c}+\xi_{v}}{2}$, and $\tau \rightarrow \tau_{3}$. Apart from the constant and the electron-hole asymmetry terms proportional to $\sigma_{0}$, there is also a trigonal warping term proportional to $\zeta$, as well as the massive Dirac Hamiltonian with a quadratic term. Estimates for $\mathrm{MoSe}_{2}$ give [16] $v=5.6 \times 10^{5} \mathrm{~ms}^{-1}, 2 \Delta=1.44 \mathrm{eV}, \delta \xi=-0.30 \mathrm{eV}, \zeta=9.4 \mathrm{meV}$, and $\xi=0.8 \mathrm{meV}$.

To obtain a low-energy two-band model for the $y>0$ side of the TGB [see Fig. 1(a)], we must recognize that the two sides are related by a $y \rightarrow-y$ transformation. This allows us to right the three-band TB Hamiltonian for $y>0$ exactly as in Eqs. (A1)-(A3), with the replacement $\beta \rightarrow-\beta$ in Eqs. (A3). It should also be noted that the $y \rightarrow-y$ transformation affects 
the atomic orbital basis $\left(d_{x y} \rightarrow-d_{x y}\right)$, so that the three component operator $\hat{\psi}_{k}^{\dagger}$ in Eq. (A1) is to be read on the $y>0$ side as $\hat{\psi}_{\boldsymbol{k}}^{\dagger}=\left(\hat{c}_{\boldsymbol{k}, z^{2}}^{\dagger}, \hat{c}_{\boldsymbol{k},-x y}^{\dagger}, \hat{c}_{\boldsymbol{k}, x^{2}-y^{2}}^{\dagger}\right)$.

We want to compare the two sides of the TGB, so it is convenient to use the same basis, which requires the transformation $\left(\hat{c}_{\boldsymbol{k}, z^{2}}^{\dagger}, \hat{c}_{\boldsymbol{k},-x y}^{\dagger}, \hat{c}_{\boldsymbol{k}, x^{2}-y^{2}}^{\dagger}\right) \rightarrow\left(\hat{c}_{\boldsymbol{k}, z^{2}}^{\dagger}, \hat{c}_{\boldsymbol{k}, x y}^{\dagger}, \hat{c}_{\boldsymbol{k}, x^{2}-y^{2}}^{\dagger}\right)$ on the $y>0$ side. The unitary operator transforming between the two basis is just $U=\operatorname{diag}(1,-1,1)$, and the transformed Hamiltonian, Taylor expanded to the second order near the $K(\tau=+1)$ and $K^{\prime}$ $(\tau=-1)$ points, reads

$$
\begin{aligned}
H(\tau \mathbf{K}+\mathbf{q}) & =H(\tau \mathbf{K})+H_{\mathbf{q}}^{(1)}+H_{\mathbf{q}}^{(2)}+\mathcal{O}(a q)^{2} \\
& =H(\tau \mathbf{K})+q_{i}\left(\partial_{i} H\right)_{\tau \mathbf{K}}+\frac{1}{2} q_{i} q_{j}\left(\partial_{i} \partial_{j} H\right)_{\tau \mathbf{K}}+\mathcal{O}(a q)^{3} \\
& =\left(\begin{array}{ccc}
\eta_{0} & -\eta_{1} & \eta_{2} \\
-\eta_{1}^{*} & \eta_{11} & -\eta_{12} \\
\eta_{2}^{*} & -\eta_{12}^{*} & \eta_{22}
\end{array}\right)+a\left(\begin{array}{ccc}
u_{0} & -u_{1} & u_{2} \\
-u_{1}^{*} & u_{11} & -u_{12} \\
u_{2}^{*} & -u_{12}^{*} & u_{22}
\end{array}\right)+a^{2}\left(\begin{array}{ccc}
v_{0} & -v_{1} & v_{2} \\
-v_{1}^{*} & v_{11} & -v_{12} \\
v_{2}^{*} & -v_{12}^{*} & v_{22}
\end{array}\right)+\mathcal{O}(a q)^{3},
\end{aligned}
$$

where the matrix elements $\eta, u$, and $v$, are the same as in Eqs. (A6)-(A8), respectively, with the replacement $q_{y} \rightarrow-q_{y}$ in Eqs. (A7) and (A8).

Diagonalizing the zeroth order Hamiltonian in Eq. (A17),

$$
H(\tau \mathbf{K})=\left(\begin{array}{ccc}
\epsilon_{1}-3 t_{0} & 0 & 0 \\
0 & \epsilon_{2}-\frac{1}{2}\left(3 t_{11}+3 t_{22}\right) & +i \tau 3 \sqrt{3} t_{12} \\
0 & -i \tau 3 \sqrt{3} t_{12} & \epsilon_{2}-\frac{1}{2}\left(3 t_{11}+3 t_{22}\right)
\end{array}\right),
$$

one obtains

$$
\begin{aligned}
\left|\psi_{c}(\tau \mathbf{K})\right\rangle & =\left|\tau \mathbf{K}, d_{z^{2}}\right\rangle, \quad \epsilon_{c}=\epsilon_{1}-3 t_{0}, \quad\left|\psi_{h}(\tau \mathbf{K})\right\rangle=\frac{1}{\sqrt{2}}\left(\left|\tau \mathbf{K}, d_{x^{2}-y^{2}}\right\rangle+i \tau\left|\tau \mathbf{K}, d_{x y}\right\rangle\right), \\
\epsilon_{h} & =\epsilon_{2}-\frac{1}{2}\left(3 t_{11}+3 t_{22}\right)+3 \sqrt{3} t_{12}, \quad\left|\psi_{v}(\tau \mathbf{K})\right\rangle=\frac{1}{\sqrt{2}}\left(\left|\tau \mathbf{K}, d_{x^{2}-y^{2}}\right\rangle-i \tau\left|\tau \mathbf{K}, d_{x y}\right\rangle\right), \\
\epsilon_{v} & =\epsilon_{2}-\frac{1}{2}\left(3 t_{11}+3 t_{22}\right)-3 \sqrt{3} t_{12} .
\end{aligned}
$$

Comparing the atomic content of the two states $\left|\psi_{v}(\tau \mathbf{K})\right\rangle$ and $\left|\psi_{h}(\tau \mathbf{K})\right\rangle$ in Eq. (A19) with their counterparts in Eq. (A10), it is apparent that a gap inversion occurs between the two as we cross the boundary. This gap inversion is further discussed in the main text, Sec. III C.

The effective Hamiltonian for $y>0$ in the subspace of the conduction and valence bands may now be obtained in a similar way to the $y<0$ side. We first use the basis in Eq. (A19) to write the expanded Hamiltonian of Eq. (A17), and then apply exactly the same procedure as for the $y<0$ side after Eq. (A10). We finally arrive at

$$
H_{\mathrm{eff}}^{(y>0)}(\mathbf{q})=v \hbar\left(q_{x} \tau_{3} \sigma_{x}-q_{y} \sigma_{y}\right)+\left(\Delta+\delta \xi a^{2} q^{2}\right) \sigma_{3}+\zeta a^{2}\left(q_{x} \tau_{3} \sigma_{x}+q_{y} \sigma_{y}\right) \sigma_{x}\left(q_{x} \tau_{3} \sigma_{x}+q_{y} \sigma_{y}\right)+\left(\epsilon_{F}+\xi q^{2}\right) \sigma_{0},
$$

which is exactly the same as Eq. (A16) after the transformation $q_{y} \rightarrow-q_{y}$. The parameters in Eq. (A20) are the same as in Eq. (A16).

\section{APPENDIX B: SOME MQIM-HO USEFUL EXPRESSIONS}

The spectra of the $\gamma=s, c, c^{\prime}$ branch lines in the spectral-function expression, Eqs. (10), are given by

$$
\begin{aligned}
& \tilde{\omega}_{s}(k)=\tilde{\varepsilon}_{s}(k)=\varepsilon_{s}(k) \leqslant 0 \text { for } k=-q^{\prime} \in\left[-k_{F}, k_{F}\right], \\
& \tilde{\omega}_{c}(k)=\tilde{\varepsilon}_{c}\left(|k|+k_{F}\right) \leqslant 0 \text { for } k=k_{c}=-\operatorname{sgn}\{k\} k_{F}-q \in\left[-k_{F}, k_{F}\right], \\
& \tilde{\omega}_{c^{\prime}}(k)=\tilde{\varepsilon}_{c}\left(|k|-k_{F}\right) \leqslant 0 \text { for } k=k_{c^{\prime}}=\operatorname{sgn}\{k\} k_{F}-q \in\left[-3 k_{F}, 3 k_{F}\right],
\end{aligned}
$$

where $\tilde{\varepsilon}_{s}\left(q^{\prime}\right)$ and $\tilde{\varepsilon}_{c}(q)$ are the $s$ and $c$ particle energy dispersions, respectively, given below in Eqs. (B2). The spectra, Eqs. (B1), are plotted within the MQIM-HO in Fig. 6(a) as a function of the excitation momentum $k$ for $u=U / 4 t=0.18$, transfer integral $t=0.58 \mathrm{eV}$, and electronic density $n_{e}=2 / 3$.

As discussed in Sec. IV B, the charge particle-charge-hole mobility impurity interaction gives rise to a slight renormalization of the $c$-band energy dispersion. Within the MQIM-HO, it is estimated to lead to

$$
\left.\tilde{\varepsilon}_{c}(q)=\left(1+\beta_{c} \theta_{c}\right) \varepsilon_{c}(q) \text { for } q \in\right]-2 k_{F}, 2 k_{F}\left[, \quad \tilde{\varepsilon}_{s}\left(q^{\prime}\right)=\varepsilon_{s}\left(q^{\prime}\right) \text { for } q^{\prime} \in\right]-k_{F}, k_{F}[,
$$

where $\beta_{c}=\frac{1}{\xi_{c}}\left(1-\frac{\xi_{c}}{\sqrt{2}}\right)$ and $\theta_{c}=1$ for $\left.\tilde{\xi}_{c} \in\right] 1 / 2,1\left[\right.$ and $\theta_{c}=\left(\frac{\xi_{c}-\tilde{\xi}_{c}}{\xi_{c}-1}\right)$ for $\left.\tilde{\xi}_{c} \in\right] 1, \xi_{c}$ [. Here the $s$-band energy dispersion, which remains invariant under the universal transformation, was also given. The 1DHM dispersions $\varepsilon_{c}(q)$ and $\varepsilon_{s}\left(q^{\prime}\right)$ in Eqs. (B1) are defined by Ma et al. [4]. 
The $\gamma=c, c^{\prime}, s$ exponents $\tilde{\zeta}_{\gamma}(k)$ in the spectral function, Eqs. (10), plotted in Fig. 7 for $u=0.18, n_{e}=2 / 3$, and $l=8$ read

$$
\begin{aligned}
\tilde{\zeta}_{c}(k) & =-\frac{1}{2}+\sum_{l= \pm 1}\left(\frac{\tilde{\xi}_{c}}{4}-\tilde{\Phi}_{c, c}\left(\iota 2 k_{F}, q\right)\right)^{2}, \text { where } k=\in\left[-k_{F}+k_{F c}^{0}, k_{F}-k_{F c}^{0}\right], \\
q & =-\operatorname{sgn}\{k\} k_{F}-k \in\left[-2 k_{F}+k_{F c}^{0},-k_{F}\right], \text { and } \\
& =-\operatorname{sgn}\{k\} k_{F}-k \in\left[k_{F}, 2 k_{F}-k_{F c}^{0}\right], \\
\tilde{\zeta}_{c^{\prime}}(k) & =-\frac{1}{2}+\sum_{l= \pm 1}\left(\frac{\tilde{\xi}_{c}}{4}-\tilde{\Phi}_{c, c}\left(\iota 2 k_{F}, q\right)\right)^{2}, \text { where } k=\in\left[-3 k_{F}+k_{F c}^{0}, 3 k_{F}-k_{F c}^{0}\right], \\
q & =\operatorname{sgn}\{k\} k_{F}-k \in\left[-2 k_{F}+k_{F c}^{0}, k_{F}\right], \text { and } \\
& =\operatorname{sgn}\{k\} k_{F}-k \in\left[-k_{F}, 2 k_{F}-k_{F c}^{0}\right], \\
\tilde{\zeta}_{s}(k) & =-1+\sum_{\iota= \pm 1}\left(-\frac{\iota}{2 \tilde{\xi}_{c}}-\tilde{\Phi}_{c, s}\left(\iota 2 k_{F}, q^{\prime}\right)\right)^{2}, \text { where } k \in\left[-k_{F}+k_{F s}^{0}, k_{F}-k_{F s}^{0}\right], \\
q^{\prime} & =-k \in\left[-k_{F}+k_{F s}^{0}, k_{F}-k_{F s}^{0}\right] .
\end{aligned}
$$

The renormalization of the phase shifts $2 \pi \tilde{\Phi}_{c, s}\left( \pm 2 k_{F}, q^{\prime}\right)$ and $2 \pi \tilde{\Phi}_{c, c}\left( \pm 2 k_{F}, q\right)$ appearing in the exponents expressions, Eqs. (B3), under the $\xi_{c} \rightarrow \tilde{\xi}_{c}$ transformation of Ma et al. [4] leads to

$$
\begin{aligned}
2 \pi \tilde{\Phi}_{c, s}\left( \pm 2 k_{F}, q^{\prime}\right) & =\frac{\tilde{\xi}_{c}}{\xi_{c}} 2 \pi \Phi_{c, s}\left( \pm 2 k_{F}, q^{\prime}\right), \quad 2 \pi \tilde{\Phi}_{c, c}\left( \pm 2 k_{F}, q\right)=2 \pi \tilde{\Phi}_{c, c}^{\tilde{a}}\left( \pm 2 k_{F}, q\right)+2 \pi \tilde{\Phi}_{c, c}^{R_{\text {eff }}}\left(k_{r}\right), \\
2 \pi \tilde{\Phi}_{c, c}^{\tilde{a}}\left( \pm 2 k_{F}, q\right) & =\frac{\xi_{c}}{\tilde{\xi}_{c}} \frac{\left(\tilde{\xi}_{c}-1\right)^{2}}{\left(\xi_{c}-1\right)^{2}} 2 \pi \Phi_{c, c}\left( \pm 2 k_{F}, q\right),
\end{aligned}
$$

for $q^{\prime} \in\left[-k_{F}, k_{F}\right], q \in\left[-2 k_{F}^{+}, 2 k_{F}^{-}\right]$, and $\left|k_{r}\right|=\left|q \mp 2 k_{F}\right| \in\left[0,4 k_{F}\right.$ [. Here $k_{r}^{0}=2 \pi / L$ and $k_{r}=\left(q \mp 2 k_{F}\right)$ are the relative momentum of the charge particle at the $c$-band Fermi points $\pm 2 k_{F}$ and charge-hole mobile impurity of $c$-band momentum $q \in\left[-2 k_{F}^{+}, 2 k_{F}^{-}\right]$and $\Phi_{c, s}\left( \pm 2 k_{F}, q^{\prime}\right)$ and $\Phi_{c, c}\left( \pm 2 k_{F}, q\right)$ are 1DHM phase shifts.

The spin-particle phase shifts remain invariant under the MQIR-LR transformation and are given by

$$
\begin{aligned}
& \tilde{\Phi}_{s, s}\left(\iota k_{F}, q^{\prime}\right)=\frac{\iota\left(\xi_{s}-1\right)\left(\xi_{s}+(-1)^{\delta_{q, k_{F}}}\right)}{2 \xi_{s}} \text { for } q^{\prime} \in\left[-k_{F}, k_{F}\right], \\
& \tilde{\Phi}_{s, c}\left(\iota k_{F}, q\right)=-\frac{\iota \xi_{s}}{4} \text { for } q \in\left[-2 k_{F}, 2 k_{F}\right],
\end{aligned}
$$

where $\xi_{s}=\sqrt{2}$ and $\iota= \pm 1$.

[1] H. Zhang, C. X. Liu, S. Gazibegovic, D. Xu, J. A. Logan, G. Wang, N. Van Loo, J. D. Bommer, M. W. De Moor, D. Car, R. L. Op Het Veld, P. J. Van Veldhoven, S. Koelling, M. A. Verheijen, M. Pendharkar, D. J. Pennachio, B. Shojaei, J. S. Lee, C. J. Palmstrøm, E. P. Bakkers, S. D. Sarma, and L. P. Kouwenhoven, Nature 556, 74 (2018).

[2] C. Blumenstein, J. Schäfer, S. Mietke, S. Meyer, A. Dollinger, M. Lochner, X. Y. Cui, L. Patthey, R. Matzdorf, and R. Claessen, Nat. Phys. 7, 776 (2011).

[3] A. Imambekov, T. L. Schmidt, and L. I. Glazman, Rev. Mod. Phys. 84, 1253 (2012).

[4] Y. Ma, H. C. Diaz, J. Avila, C. Chen, V. Kalappattil, R. Das, M.-H. Phan, T. Čadež, J. M. P. Carmelo, M. C. Asensio, and M. Batzill, Nat. Commun. 8, 14231 (2017).

[5] X. He, H. Htoon, S. K. Doorn, W. H. P. Pernice, F. Pyatkov, R. Krupke, A. Jeantet, Y. Chassagneux, and C. Voisin, Nat. Mater. 17, 663 (2018).
[6] V. Mourik, K. Zuo, S. M. Frolov, S. R. Plissard, E. P. A. M. Bakkers, and L. P. Kouwenhoven, Science 336, 1003 (2012).

[7] S. Nadj-Perge, I. K. Drozdov, J. Li, H. Chen, S. Jeon, J. Seo, A. H. MacDonald, B. A. Bernevig, and A. Yazdani, Science 346, 602 (2014).

[8] A. H. Castro Neto, F. Guinea, N. M. R. Peres, K. S. Novoselov, and A. K. Geim, Rev. Mod. Phys. 81, 109 (2009).

[9] X. Xu, W. Yao, D. Xiao, and T. F. Heinz, Nat. Phys. 10, 343 (2014).

[10] Q. H. Wang, K. Kalantar-Zadeh, A. Kis, J. N. Coleman, and M. S. Strano, Nat. Nanotechnol. 7, 699 (2012).

[11] O. V. Y. Kis and A. Kis, Mater. Today 18, 20 (2015).

[12] A. M. Van Der Zande, P. Y. Huang, D. A. Chenet, T. C. Berkelbach, Y. You, G. H. Lee, T. F. Heinz, D. R. Reichman, D. A. Muller, and J. C. Hone, Nat. Mater. 12, 554 (2013).

[13] H. Liu, L. Jiao, F. Yang, Y. Cai, X. Wu, W. Ho, C. Gao, J. Jia, N. Wang, H. Fan, W. Yao, and M. Xie, Phys. Rev. Lett. 113, 066105 (2014). 
[14] S. Barja, S. Wickenburg, Z. F. Liu, Y. Zhang, H. Ryu, M. M. Ugeda, Z. Hussain, Z. X. Shen, S. K. Mo, E. Wong, M. B. Salmeron, F. Wang, M. F. Crommie, D. F. Ogletree, J. B. Neaton, and A. Weber-Bargioni, Nat. Phys. 12, 751 (2016).

[15] Y. Ma, S. Kolekar, H. Coy Diaz, J. Aprojanz, I. Miccoli, C. Tegenkamp, and M. Batzill, ACS Nano 11, 5130 (2017).

[16] G.-B. Liu, W.-Y. Shan, Y. Yao, W. Yao, and D. Xiao, Phys. Rev. B 88, 085433 (2013).

[17] X. Zou, Y. Liu, and B. I. Yakobson, Nano Lett. 13, 253 (2013).

[18] O. Lehtinen, H.-P. Komsa, A. Pulkin, M. B. Whitwick, M.-W. Chen, T. Lehnert, M. J. Mohn, O. V. Yazyev, A. Kis, U. Kaiser, and A. V. Krasheninnikov, ACS Nano 9, 3274 (2015).

[19] B. Andrei Bernevig with Taylor L. Hughes, Topological Insulators and Topological Superconductors (Princeton University Press, New Jersey, 2013).

[20] L. Zhu, E. Prodan, and K. H. Ahn, Phys. Rev. B 99, 041117 (2019).

[21] We use the hopping integrals obtained from the GGA DFT calculation of Ref. [16].

[22] M. V. Bollinger, J. V. Lauritsen, K. W. Jacobsen, J. K. Nørskov, S. Helveg, and F. Besenbacher, Phys. Rev. Lett. 87, 196803 (2001).

[23] Y. Li, Z. Zhou, S. Zhang, and Z. Chen, J. Am. Chem. Soc. 130, 16739 (2008).

[24] R.-L. Chu, G.-B. Liu, W. Yao, X. Xu, D. Xiao, and C. Zhang, Phys. Rev. B 89, 155317 (2014).

[25] L. Li, E. V. Castro, and P. D. Sacramento, Phys. Rev. B 94, 195419 (2016).

[26] D. Le and T. S. Rahman, J. Phys.: Condens. Matter 25, 312201 (2013).

[27] D. Xiao, G.-B. Liu, W. Feng, X. Xu, and W. Yao, Phys. Rev. Lett. 108, 196802 (2012).

[28] E. Cappelluti, R. Roldán, J. A. Silva-Guillén, P. Ordejón, and F. Guinea, Phys. Rev. B 88, 075409 (2013).
[29] H. Rostami, A. G. Moghaddam, and R. Asgari, Phys. Rev. B 88, 085440 (2013).

[30] D. Xiao, M. C. Chang, and Q. Niu, Rev. Mod. Phys. 82, 1959 (2010).

[31] C.-K. K. Chiu, J. C. Y. Teo, A. P. Schnyder, and S. Ryu, Rev. Mod. Phys. 88, 035005 (2016).

[32] H. Z. Lu, W. Y. Shan, W. Yao, Q. Niu, and S. Q. Shen, Phys. Rev. B 81, 115407 (2010).

[33] S.-Q. Shen, Topological Insulators, Springer Series in Solid-State Sciences, Vol. 174 (Springer, Berlin, 2012), p. 23.

[34] H. Rostami, R. Asgari, and F. Guinea, J. Phys.: Condens. Matter 28, 495001 (2016).

[35] I. Martin, Y. M. Blanter, and A. F. Morpurgo, Phys. Rev. Lett. 100, 036804 (2008).

[36] F. Zhang, A. H. MacDonald, and E. J. Mele, Proc. Natl. Acad. Sci. USA 110, 10546 (2013).

[37] A. Vaezi, Y. Liang, D. H. Ngai, L. Yang, and E. A. Kim, Phys. Rev. X 3, 021018 (2013).

[38] M. A. N. Araújo and E. V. Castro, J. Phys.: Condens. Matter 26, 075501 (2014).

[39] R.-J. Slager, V. Juričić, V. Lahtinen, and J. Zaanen, Phys. Rev. B 93, 245406 (2016).

[40] H. Guo and S. Chen, Phys. Rev. B 91, 041402 (2015).

[41] Y. Ohtsubo, J.-i. Kishi, K. Hagiwara, P. Le Fèvre, F. Bertran, A. Taleb-Ibrahimi, H. Yamane, S.-i. Ideta, M. Matsunami, K. Tanaka, and S.-i. Kimura, Phys. Rev. Lett. 115, 256404 (2015).

[42] H. J. Schulz, Phys. Rev. Lett. 64, 2831 (1990).

[43] X.-G. Wen, Rev. Mod. Phys. 89, 041004 (2017).

[44] J. Carmelo and P. Sacramento, Phys. Rep. 749, 1 (2018).

[45] J. M. P. Carmelo, T. Čadež, Y. Ohtsubo, S.-i. Kimura, and D. K. Campbell, arXiv:1801.07732.

[46] M. Hohenadler, S. Wessel, M. Daghofer, and F. F. Assaad, Phys. Rev. B 85, 195115 (2012). 